\title{
Anti-nonspecific adsorption segments-assisted self-driven surface imprinted fibers for efficient protein separation
}

\author{
Zuoting Yang ${ }^{1}$, Ting Wang ${ }^{1}$, Yabin Wang ${ }^{1}$, Qiuyu Zhang ${ }^{1}$, and Baoliang Zhang ${ }^{1}$ \\ ${ }^{1}$ Northwestern Polytechnical University
}

December 8, 2021

\begin{abstract}
At present, the development of high-performance protein imprinted materials is still a research hotspot in the field of protein imprinting. Herein, anti-protein adsorption segment (CBMA)-assisted self-driven BSA surface imprinted fibers MTCFs@SIP@CBMA with high recognition selectivity are pioneered using the strategies of combining magnetic nanomaterial surface imprinting technique with amino-Michael addition. The special structure of the carrier MTCFs endows MTCFs@SIP@CBMA with magnetic performance and self-driven adsorption performance, which simplifies the separation process while improving the adsorption capacity and accelerating the adsorption rate. The adsorption capacity for BSA reached $395.26 \mathrm{mg} / \mathrm{g}$ within $30 \mathrm{~min}$. The introduction of CBMA segments on the surface after imprinting by amino-Michael addition makes its polymer chain length and position controllable. Under the strongest anti-nonspecific adsorption effect, MTCFs@SIP@CBMA exhibit excellent specific identification to BSA from mixed proteins. Additionally, MTCFs@SIP@CBMA show considerable reusability. Therefore, MTCFs@SIP@CBMA are expected to be applied in efficient separation of proteins in biological samples.
\end{abstract}

\section{Anti-nonspecific adsorption segments-assisted self-driven surface imprinted fibers for efficient protein separation}

Zuoting Yang ${ }^{1,2}$, Ting Wang ${ }^{1}$, Yabin Wang ${ }^{1,3}$, Qiuyu Zhang ${ }^{1,2} 11^{*}$ Corresponding authors:, Baoliang Zhang ${ }^{1,4}$ 22Northwestern Polytechnical University, Youyi Road 127\#, Xi'an (710072), China. Email: qyzhang@nwpu.edu.cn, blzhang@nwpu.edu.cn

${ }^{1}$ School of Chemistry and Chemical Engineering, Northwestern Polytechnical University, Xi'an, 710129, P. R. China

${ }^{2}$ Xi'an Key Laboratory of Functional Organic Porous Materials, Northwestern Polytechnical University, Xi'an, 710072, P. R. China

${ }^{3}$ Shaanxi Key Laboratory of Chemical Reaction Engineering, College of Chemistry and Chemical Engineering, Yan'an University, Yan'an 716000, PR China

${ }^{4}$ Shaanxi Engineering and Research Center for Functional Polymers on Adsorption and Separation, Sunresins New Materials Co. Ltd., Xi'an, 710072, China

Abstract: At present, the development of high-performance protein imprinted materials is still a research hotspot in the field of protein imprinting. Herein, anti-protein adsorption segment (CBMA)-assisted selfdriven BSA surface imprinted fibers MTCFs@SIP@CBMA with high recognition selectivity are pioneered using the strategies of combining magnetic nanomaterial surface imprinting technique with amino-Michael addition. The special structure of the carrier MTCFs endows MTCFs@SIP@CBMA with magnetic performance and self-driven adsorption performance, which simplifies the separation process while improving the adsorption capacity and accelerating the adsorption rate. The adsorption capacity for BSA reached 395.26 $\mathrm{mg} / \mathrm{g}$ within $30 \mathrm{~min}$. The introduction of CBMA segments on the surface after imprinting by amino-Michael 
addition makes its polymer chain length and position controllable. Under the strongest anti-nonspecific adsorption effect, MTCFs@SIP@CBMA exhibit excellent specific identification to BSA from mixed proteins. Additionally, MTCFs@SIP@CBMA show considerable reusability. Therefore, MTCFs@SIP@CBMA are expected to be applied in efficient separation of proteins in biological samples.

Keywords: protein imprinted materials; magnetic tubular nanofiber; anti-protein adsorption; self-driven adsorption; high recognition selectivity

\section{Introduction}

The highly selective recognition of proteins provides important technical support for the development of protein drugs, medical diagnosis, and the exploration of the pathogenesis of major diseases based on proteomics. It is an important research topic in the fields of life sciences and biotechnology ${ }^{1,2}$. Therefore, the separation, purification and detection of proteins are particularly important. As a new type of protein identification and separation material, protein imprinted polymers have the advantages of easy preparation, low cost, high selectivity, strong practicability and promising mechanical stability, so it is widely favored by researchers ${ }^{3-5}$. After a prolonged endeavor, in recent years, the imprinting methods for proteins and other types of macromolecular templates have made some research progress ${ }^{6-9}$. However, compared with imprinted polymers based on small molecules as templates, the recognition selectivity, separation rate and separation amount of protein imprinted polymers are difficult to meet the needs of practical applications. This is due to the large molecular volume, complex structure and flexible conformation of proteins ${ }^{10-12}$. In view of the specificity of protein structure, researchers have developed different strategies for protein imprinting, including epitope imprinting ${ }^{13-15}$, surface imprinting ${ }^{16-18}$, the introduction of stimulus-responsive functional monomers or cross-linking agents ${ }^{19-21}$ and anti-protein adsorption segments ${ }^{22-24}$, etc.

At present, among many protein imprinting techniques, surface imprinting based on micro/nano materials is a popular and most suitable method for preparing protein imprinted polymers. Surface imprinting refers to coating an ultra-thin polymer layer containing template molecules on the surface of the micro/nano carrier materials. The imprinting sites are located near the surface of the outer shell layer, making it easier for proteins to enter and exit the recognition sites of the polymers. Therefore, it is an effective way to overcome the mass transfer difficulties caused by the large molecular size of the protein and increase the adsorption amount of the template proteins. For surface protein imprinting, the choice of carrier is crucial. Magnetic micro/nano materials can move in a controlled direction under an external magnetic field, avoiding tedious sample handling and separation processes. So, they have been widely used in many fields ${ }^{25-27}$. Based on this advantage, protein imprinting also began to develop magnetic micro/nano materials as polymeric carriers. A series of protein imprinting was carried out on their surfaces to obtain magnetic surface imprinted polymers with easy separation and good adsorption performance ${ }^{28-31}$. For example, our research group previously prepared surface imprinted magnetic graphene $\left(\mathrm{Fe}_{3} \mathrm{O}_{4} @ \mathrm{rGO} @ \mathrm{MIPs}\right)$ microspheres ${ }^{32}$ and surface imprinted nitrogen-doped magnetic carbon nanotubes (N-MCNTs@MIPs) ${ }^{33}$ by imprinting bovine serum protein (BSA) on the surface of magnetic graphene microspheres $\left(\mathrm{Fe}_{3} \mathrm{O}_{4} @ \mathrm{gGO}\right)$ and nitrogen doped magnetic carbon nanotubes (N-MCNTS). The two imprinted materials could achieve rapid separation under the external magnetic field, and show rapid binding ability and high adsorption capacity. The adsorption capacity of BSA was $317.58 \mathrm{mg} / \mathrm{g}$ and $150.86 \mathrm{mg} / \mathrm{g}$, respectively. In addition, considering the complex structure of the protein and a variety of amino acid residues rich on its surface, it is inevitable that the protein will interact with the material surface outside the imprinted sites, resulting in non-specific adsorption. Consequently, the specific recognition ability of the imprinted materials is reduced. Therefore, how to suppress non-specific adsorption is also an urgent problem in the field of protein imprinting. Studies have proved that amphoteric polymers could form a hydration layer on their surface through electrostatic interaction or hydrogen bonding with water molecules, which significantly reduces the adsorption of proteins on the surface of materials ${ }^{34,35}$. Moreover, the amphoteric polymers possess a charged positive and negative charge center, which would not change the spatial conformation of the binding proteins. The super-hydrophilic characteristics determine that the coupling protein could still maintain its own activity. Therefore, introducing amphoteric polymers with anti-protein adsorption properties into the imprinting shell is an ideal 
method for preparing protein imprinted materials with high specific recognition ability. For instance, Wang et al. ${ }^{36}$ introduced sultaine methacrylate (SBMA) as an anti-protein adsorption chain into the imprinted shell, and successfully prepared BSA imprinted magnetic microspheres $\left(\mathrm{Fe}_{3} \mathrm{O}_{4} @ \mathrm{SiO}_{2} @ M I P\right)$ by precipitation copolymerization. The imprinted microspheres showed high adsorption capacity ( $Q_{M I P}=116.39 \mathrm{mg} / \mathrm{g}$ ) and excellent selectivity $(\mathrm{IF}=4.73)$ for BSA. However, most of the reported methods for introducing anti-protein adsorption segments are directly copolymerizing them with functional monomers ${ }^{36-38}$. It is difficult to ensure that all anti-protein components are not present in the imprinting sites, which is prone to reduce the amounts of imprinted sites. As a result, the adsorption capacity is reduced. Additionally, the length and arrangement of the anti-protein adsorption chain segments in the imprinted layer cannot be precisely controlled. Based on the above reasons, it is urgent to develop a new method to introduce anti-protein adsorption polymer chains. Noteworthily, the conformational changes of the proteins during the imprinting process would also cause the reduction of the selective recognition of imprinting sites. Therefore, mild experimental conditions should be selected as far as possible to prepare the protein imprinted polymers for avoiding the conformational change of proteins during the imprinting process. Dopamine (DA) could self-polymerize on the surface of the materials under dissolved oxygen and alkaline conditions, and the polymerization conditions are very mild. It has been widely used as a functional monomer for protein imprinting ${ }^{39-41}$.

Aiming at the difficulties of low adsorption capacity, slow adsorption rate, difficult mass transfer and poor selective recognition of protein imprinted materials, this work developed an anti-protein adsorption chain 3[[2-(Methacryloxy)ethyl]dimethylammonium]propionate (CBMA)-assisted self-driven BSA surface imprinted material with high recognition selectivity (MTCFs@SIP@CBMA) by adopting the strategy of combining magnetic nanomaterial surface imprinting with amino-Michael addition. The carrier is a magnetic tubular carbon nanofiber with porous tube wall. Its special structure endows imprinted materials with magnetic properties and self-driven performance, which are beneficial to simplify the separation process, increase the adsorption capacity, and accelerate the adsorption rate. To improve the recognition selectivity of the imprinted materials, on the one hand, the protein structure-friendly biological dopamine is selected as a functional monomer to perform the coating of imprinted polymers, ensuring that the imprinting process is mild and avoiding the conformational change of proteins. On the other hand, CBMA is selected as the anti-protein adsorption chain segment to be introduced onto the imprinted polymer shell on the surface of materials. The purpose is to reduce non-specific adsorption. Different from the copolymerization method previously reported, this work adopts the method of first coating the imprinted polymers on the surface of the carriers and then grafting the anti-protein adsorption chain segments CBMA on the surface of the imprinted shell layer by amino-Michael addition. In this way, the position and length of the CBMA polymer chain are controllable, and the recognition selectivity of the imprinted materials can be improved on the premise of ensuring a high adsorption capacity. Based on the above design, the influence of the length of CBMA polymer chain on the adsorption capacity and imprinting factor of MTCFs@SIP@CBMA is investigated. In addition, the developed materials are systematically characterized, and the adsorption performance, recognition selectivity, physical separation ability and reusability of MTCFs@SIP@CBMA are simultaneously studied.

\section{Experimental section}

Synthesis of BSA surface imprinted magnetic tubular carbon nanofibers

The preparation process of BSA surface imprinted magnetic tubular carbon nanofibers (BSA-MTCFs@SIP) was divided into two steps. In the first step, the carrier magnetic tubular carbon nanofibers (MTCFs) were synthesized using the method previously reported by our research group. ${ }^{[42,43]}$ The specific preparation process was displayed in the supporting information. In the second step, the surface of MTCFs was coated with a polydopamine (PDA) imprinted shell. The typical process was as follows: $30 \mathrm{mg}$ of MTCFs and 60 $\mathrm{mg}$ of Tween 20 were ultrasonically dispersed in $20 \mathrm{~mL}$ Tris $-\mathrm{HCl}$ buffer $(\mathrm{pH}=8.5)$, and $20 \mathrm{mg}$ BSA was added. After stirring for $30 \mathrm{~min}, 10 \mathrm{~mL}$ of Tris- $\mathrm{HCl}$ buffer solution containing $30 \mathrm{mg}$ of DA was quickly added to the above dispersion. The reaction was carried out for $14 \mathrm{~h}$ at room temperature. The products were magnetically separated and washed repeatedly with ultrapure water to remove unreacted monomer and template protein, BSA-MTCFs@SIP was obtained under vacuum drying. 
2.2 Preparation of BSA imprinted magnetic carbon nanofibers with abundant CBMA polymer segments on the surface

CBMA polymer segments grafted BSA imprinted magnetic carbon nanofibers (BSA-MTCFs@SIP@CBMA) were obtained by one-step reaction of amino-Michael addition. Firstly, $30 \mathrm{mg}$ of BSA-MTCFs@SIP was dispersed ultrasonically in a three-mouth flask containing $40 \mathrm{~mL}$ water and $10 \mathrm{~mL}$ ethanol. Then, an aqueous solution with $150 \mathrm{mg}$ CBMA was dropped into the above dispersion, followed by the addition of $0.375 \mathrm{~mL}$ triethylamine. After the reaction was performed at $25^{\circ} \mathrm{C}$ for $24 \mathrm{~h}$, the products were collected by magnetic separation, washed repeatedly with ultrapure water, and dried in vacuum to obtain BSAMTCFs@SIP@CBMA.

\subsection{Elution of BSA-MTCFs@SIP@CBMA}

A certain amount of BSA-MTCFs@SIP@CBMA was dispersed in 6\% (V/V) glacial acetic acid solution and washed at 25 to remove template molecules. The absorbance of BSA in the eluent was tracked and measured at $278 \mathrm{~nm}$ by UV-vis spectrophotometer until there was no obvious absorption peak in the eluent. After washing with ultrapure water to neutral, vacuum drying was performed to obtain CBMA grafted surface imprinted magnetic tubular carbon nanofibers (MTCFs@SIP@CBMA). As a control, CBMA grafted nonimprinted magnetic carbon nanofibers (MTCFs@NIP@CBMA) were prepared using a method similar to that of MTCFs@SIP@CBMA, but without the addition of template molecule BSA.

\section{Results and discussion}

\section{Design and synthesis of MTCFs@SIP@CBMA}

In order to construct high-performance protein imprinted materials, a zwitterionic polymer CBMA-assisted self-driven BSA surface imprinted magnetic tubular carbon nanofibers MTCFs@SIP@CBMA was designed and synthesized in this work. The preparation process is designed in Figure 1. Firstly, magnetic tubular carbon nanofibers (MTCFs) were prepared by vacuum high temperature calcination using polymer nanofibers without extracting the template $\mathrm{FeCl}_{3}$ as precursor. On the one hand, MTCFs were magnetic, simplifying the separation process without the need for cumbersome, energy-consuming techniques such as filtration or centrifugation. On the other hand, the tube wall of MTCFs possessed abundant pores and contained internal cavities. This special structure gave the imprinted materials with self-driven adsorption performance. When it was dispersed in a high-concentration BSA solution, a concentration gradient was formed between the cavity inside the tube and the outside of the tube, resulting in an osmotic pressure difference. The osmotic pressure difference would drive BSA molecules to spontaneously bind to the imprinted sites on the surface, and further migrated to the internal cavity through the pores of the tube wall. Accordingly, the adsorption capacity was significantly increased and the adsorption speed was accelerated. Therefore, MTFs was chosen as the carrier to cover the imprinting layer. Utilizing BSA as template molecules and biofriendly dopamine as functional monomers, BSA-MTCFs@SIP were prepared by coating imprinted polymers on the surface of MTCFs through oxidative self-polymerization. Subsequently, to improve the recognition selectivity of the imprinted materials, the zwitterionic polymer CBMA as an anti-protein adsorption segment was grafted on the surface of the imprinting layer to reduce the non-specific adsorption of materials. Different from the copolymerization method previously reported, in this manuscript, the anti-protein adsorption chain segments were introduced by first coating the polydopamine imprinted layer on the carrier and then grafting CBMA on the surface of the imprinted layer via amino-Michael addition. Therefore, the location and length of CBMA were controllable, which can improve the recognition selectivity of imprinted materials under the premise of ensuring high adsorption capacity. After the elution step by 6\% HAc, the surface of MTCFs@SIP@CBMA produced imprinting sites with high recognition selectivity that match BSA in three-dimensional space and functional groups. 


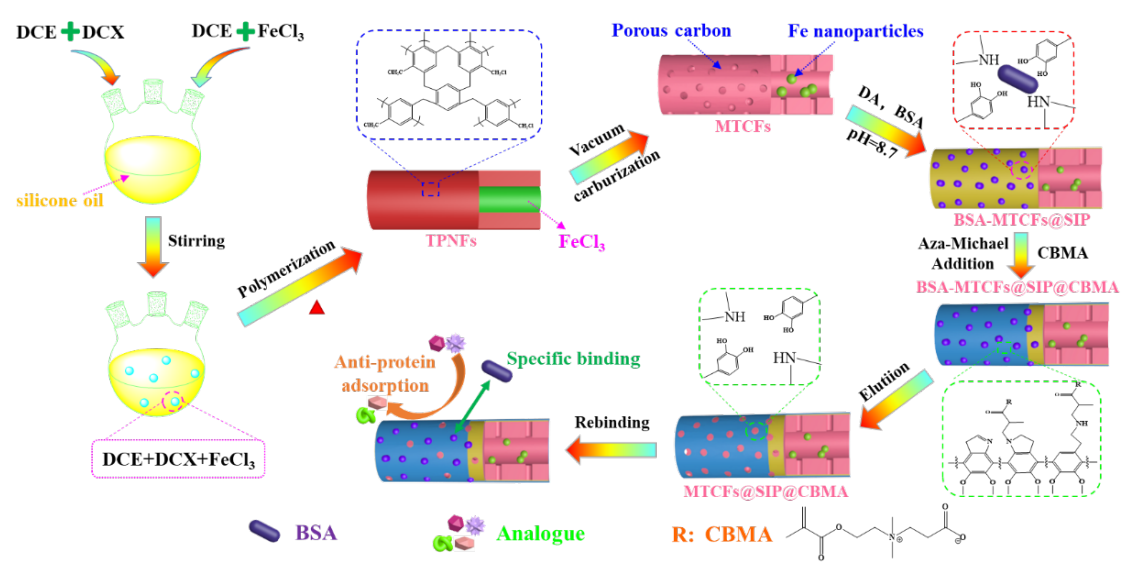

Figure 1 Synthesis schematic diagram of MTCFs@SIP@CBMA

Influence of CBMA polymer chain length on the imprinting factor of MTCFs@SIP@CBMA

The strength of the anti-protein effect depended on the length of the CBMA polymer segments. Meanwhile, the length of the CBMA polymer segments could be intuitively reflected by the thickness of the CBMA polymer layer. The length of the CBMA anti-protein adsorption chain was controlled by changing the amount of CBMA added during the amino-Michael addition process. As shown in Figure 2a, the thickness of the CBMA layer gradually increased as the dose of CBMA increased, indicating that the length of the CBMA polymer chain gradually increased. The effect of CBMA polymer chain length on the adsorption capacity and imprinting factor of MTCFs@SIP@CBMA is shown in Figure 2b. It can be seen that with the increase of CBMA polymer chain length, the adsorption capacity of MTCFs@SIP@CBMA on BSA gradually decreased, while the imprinting factor demonstrated a trend of first increasing and then decreasing. This was attributed to the weak anti-protein effect when the CBMA chain length was too short. So, the non-specific adsorption of the materials for non-template proteins cannot be effectively reduced. However, too long CBMA chains could easily lead to strong anti-protein adsorption effect and increase steric hindrance, which was not conducive to the binding of template proteins to imprinting sites. The experimental results suggested that when the mass ratio of BSA-MTCFs@SIP to CBMA was 1:10, the CBMA chain length was the most appropriate. MTCFs@SIP@CBMA possessed the highest imprinting factor for BSA. Therefore, MTCFs@SIP@CBMA prepared at this ratio was employed in the subsequent experiments. According to the results obtained, to significantly improve the recognition selectivity of imprinted materials, it was necessary to ensure that the imprinting sites were not severely affected by the CBMA anti-protein adsorption segments, while the effect of anti-protein adsorption chain segments reducing non-specific adsorption should be maximized by optimizing the chain length of CBMA polymers. 


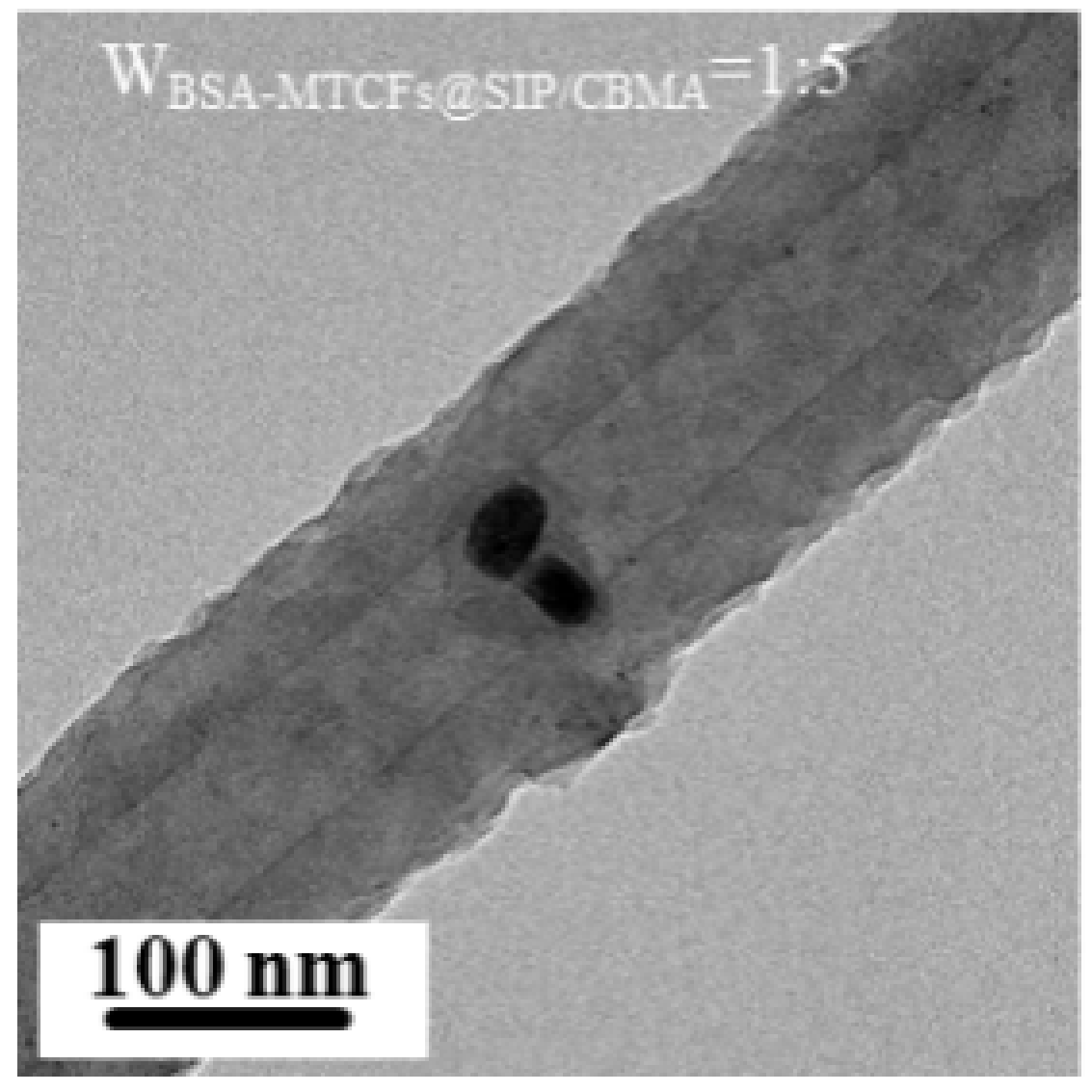




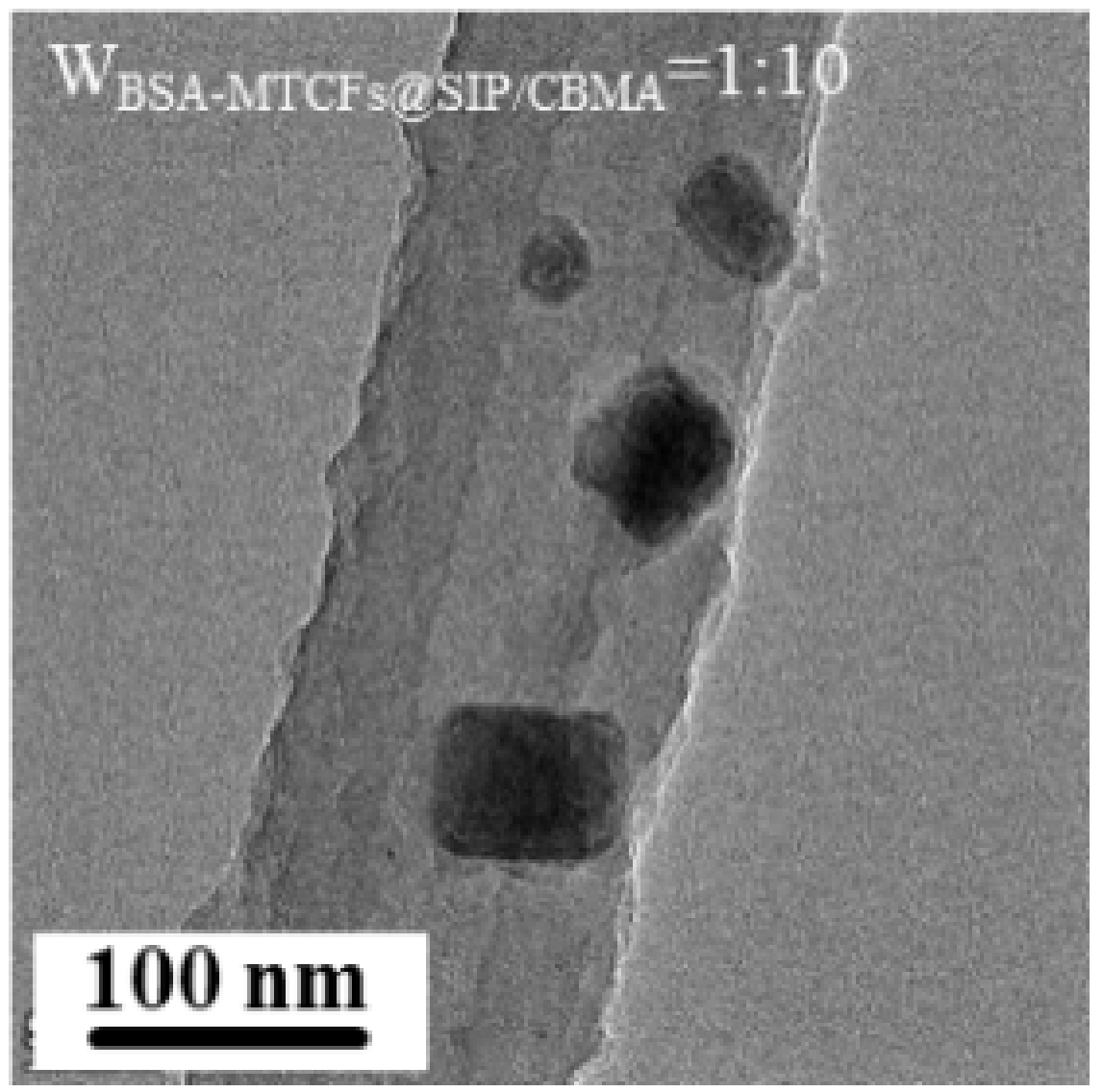




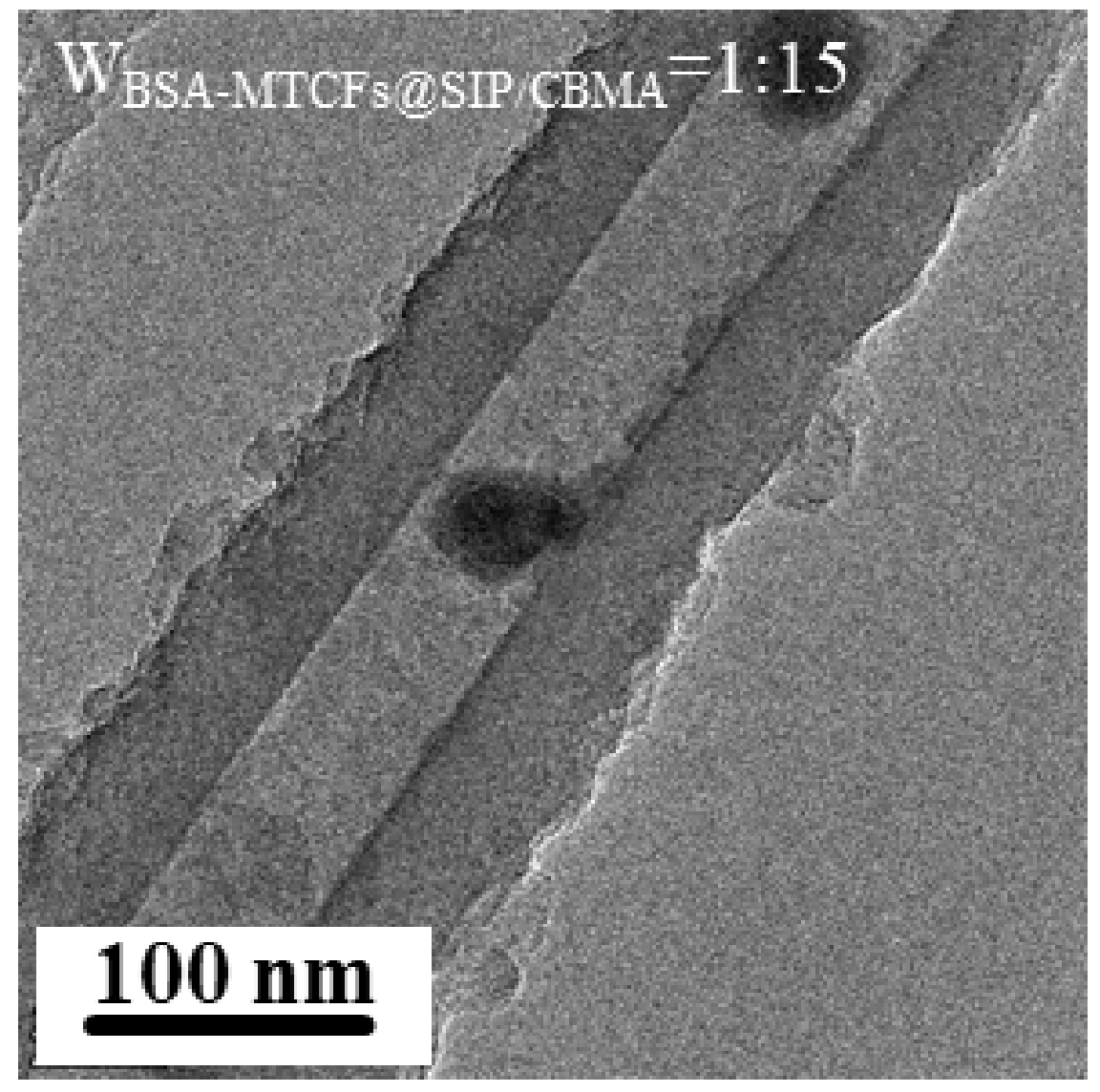




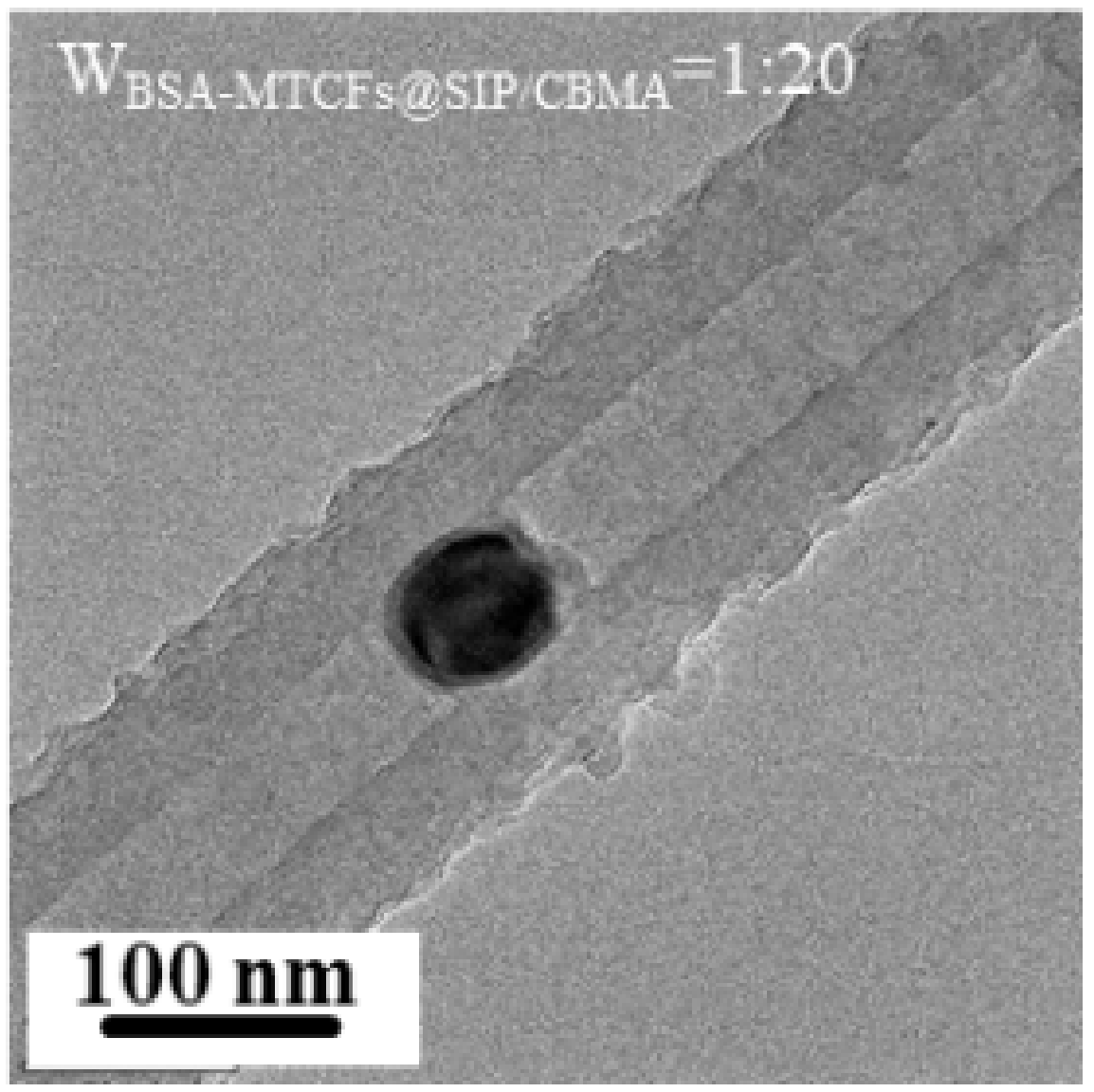

Figure 2 TEM photos of MTCFs@SIP@CBMA under different $\mathrm{W}_{\text {BSA-SIPMTFs }} / \mathrm{W}_{\text {CBMA }}$ (a); effect of $\mathrm{W}_{\mathrm{BSA} \text {-SIPMTFs }} / \mathrm{W}_{\mathrm{CBMA}}$ on the adsorption capacity and imprinting factor of MTCFs@SIP@CBMA (b)

Characterization of MTCFs@SIP@CBMA

Morphologies observation

SEM and TEM images of each product and EDS analysis results of MTCFs@SIP@CBMA are given in Figure 3. As can be seen from Figure 3A and E, MTFs presented a hollow fibrous structure with an average diameter of $105 \mathrm{~nm}$. In addition, the cavities contained black nanoscale particles. Through analysis, it was believed that these nanoparticles with high mass thick contrast were the conversion products of $\mathrm{FeCl}_{3}$ during the carbonization of polymer nanofibers (TPNFs). Magnet and VSM tests displayed that MTCFs exhibited magnetic responsiveness, so it was inferred that these nanoparticles may be $\mathrm{Fe}_{3} \mathrm{O}_{4}$ or iron element. After the coating of imprinted layer, the surface of BSA-MTCFs@SIP became significantly rough compared with the smooth surface of MTCFs, and the fiber diameter increased slightly (Figure. 3b and f). The average diameter of BSA-MTCFs@SIP was121 nm, which was an increase of $16 \mathrm{~nm}$ compared to MTCFs. The calculated thickness of imprinting layer was $8 \mathrm{~nm}$. This thickness was sufficient to achieve an effective imprinting of BSA with a size of $14 \mathrm{~nm} \times 4 \mathrm{~nm} \times 4 \mathrm{~nm}$. SEM and TEM photos of MTCFs@SIP@CBMA are presented in Figure 3c and g. By measurement, the average diameter of MTCFs@SIP@CBMA fibers increased by 4 $\mathrm{nm}$ compared with that of BSA-MTCFs@SIP, indicating the successful graft of CBMA polymer chain. The thickness of CBMA layer was $2 \mathrm{~nm}$. Compared Figures 3c with d, it was observed that the surface morphology of MTCFs@NIP@CBMA was not significantly different from that of MTCFs@SIP@CBMA. In the dark field image of MTCFs@SIP@CBMA (Figure 3h), it displayed a different degree of brightness and darkness on the tube wall, illustrating the coating of the PDA imprinted layer and the grafting of the CBMA polymer 
chain. The extremely bright nanoscale dots in the tube were inorganic magnetic nanoparticles. The EDS analysis results of MTCFs@SIP@CBMA are shown in Figure 3i-1. It can be seen that MTCFs@SIP@CBMA contained abundant $\mathrm{C}, \mathrm{N}, \mathrm{O}$ and Fe elements. The presence of $\mathrm{N}$ and $\mathrm{O}$ indicated that the surface of MTCFs was coated with PDA imprinted layer and CBMA polymer layer. Fe element was derived from magnetic nanoparticles.
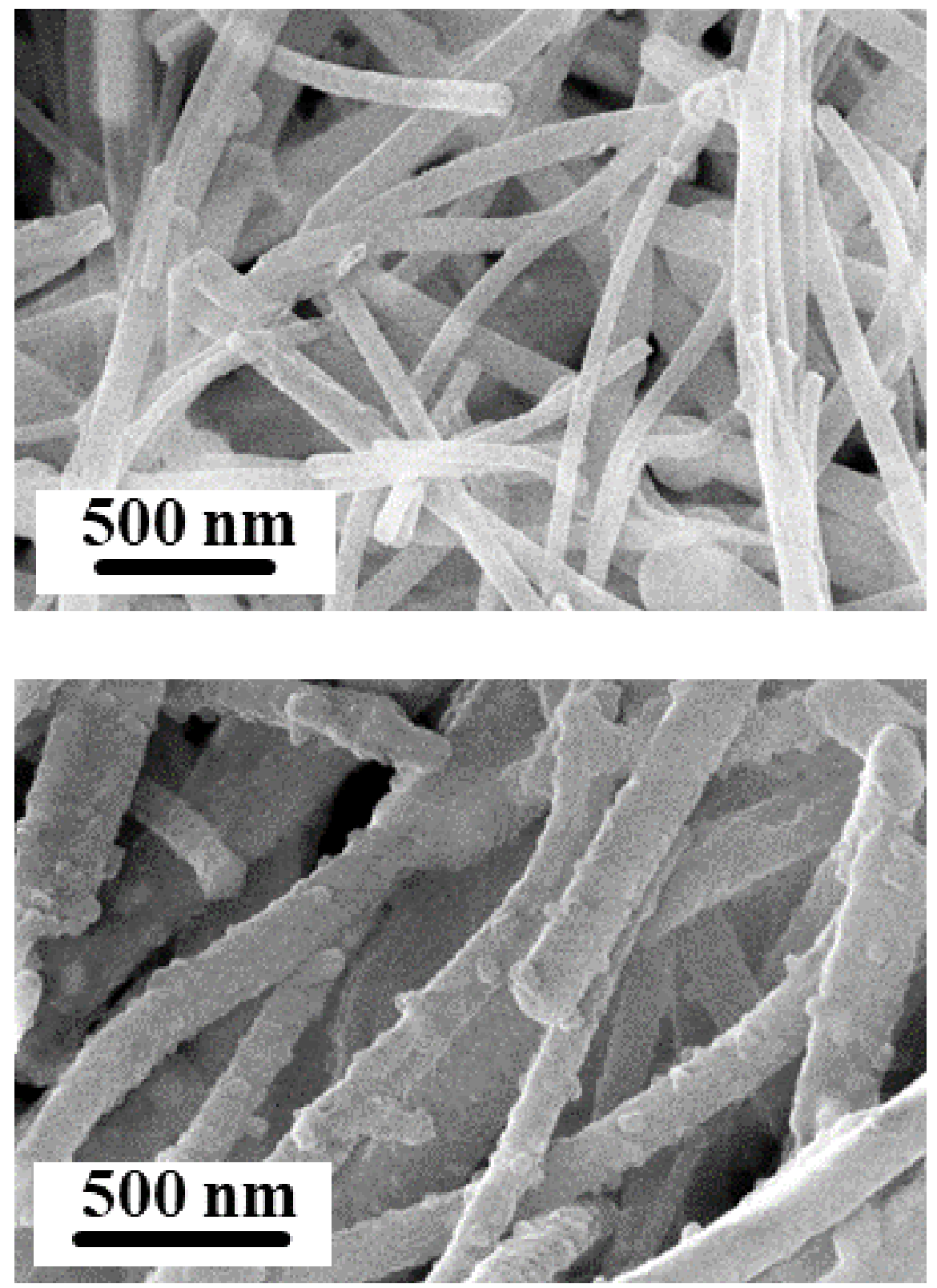

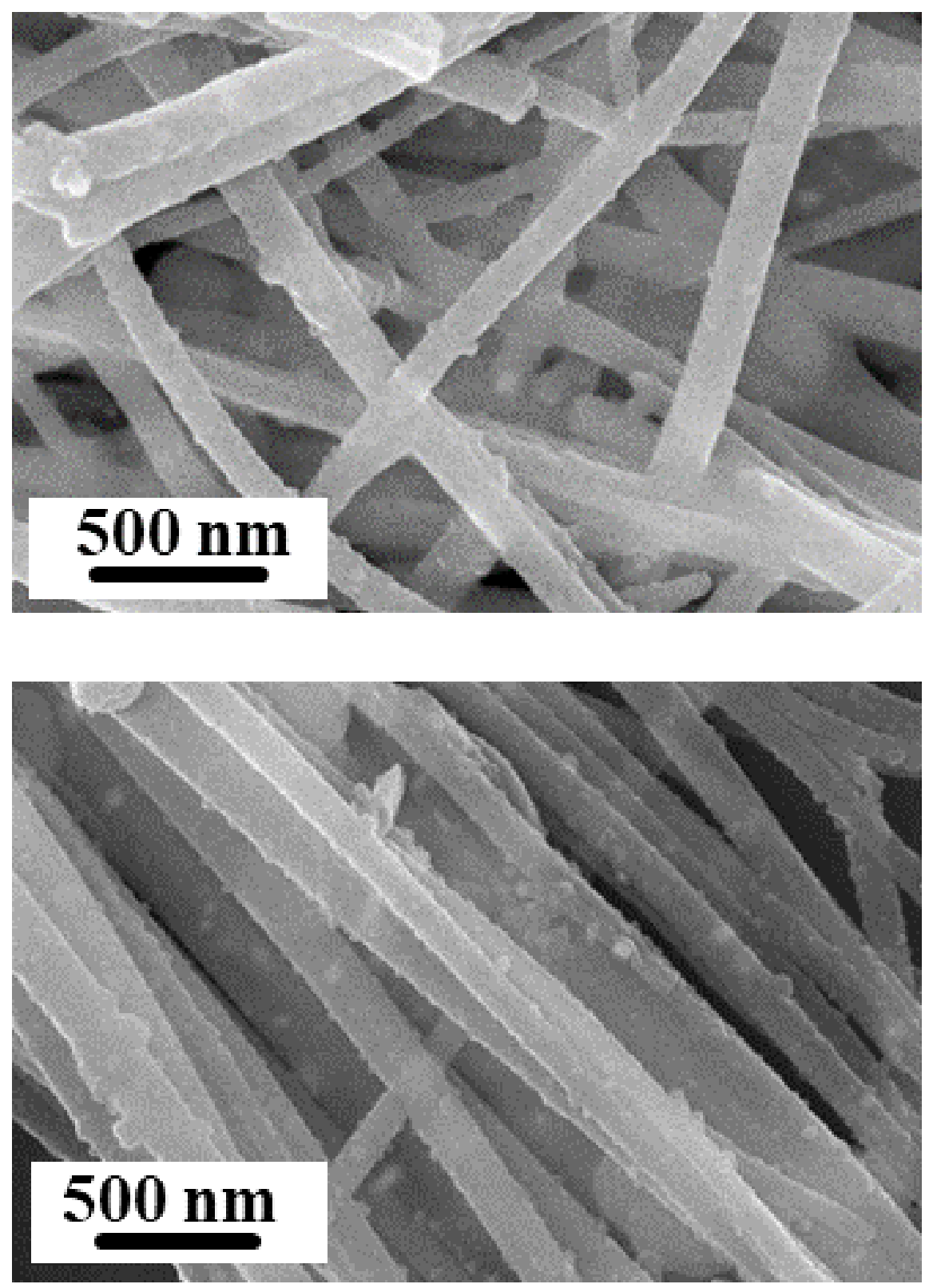


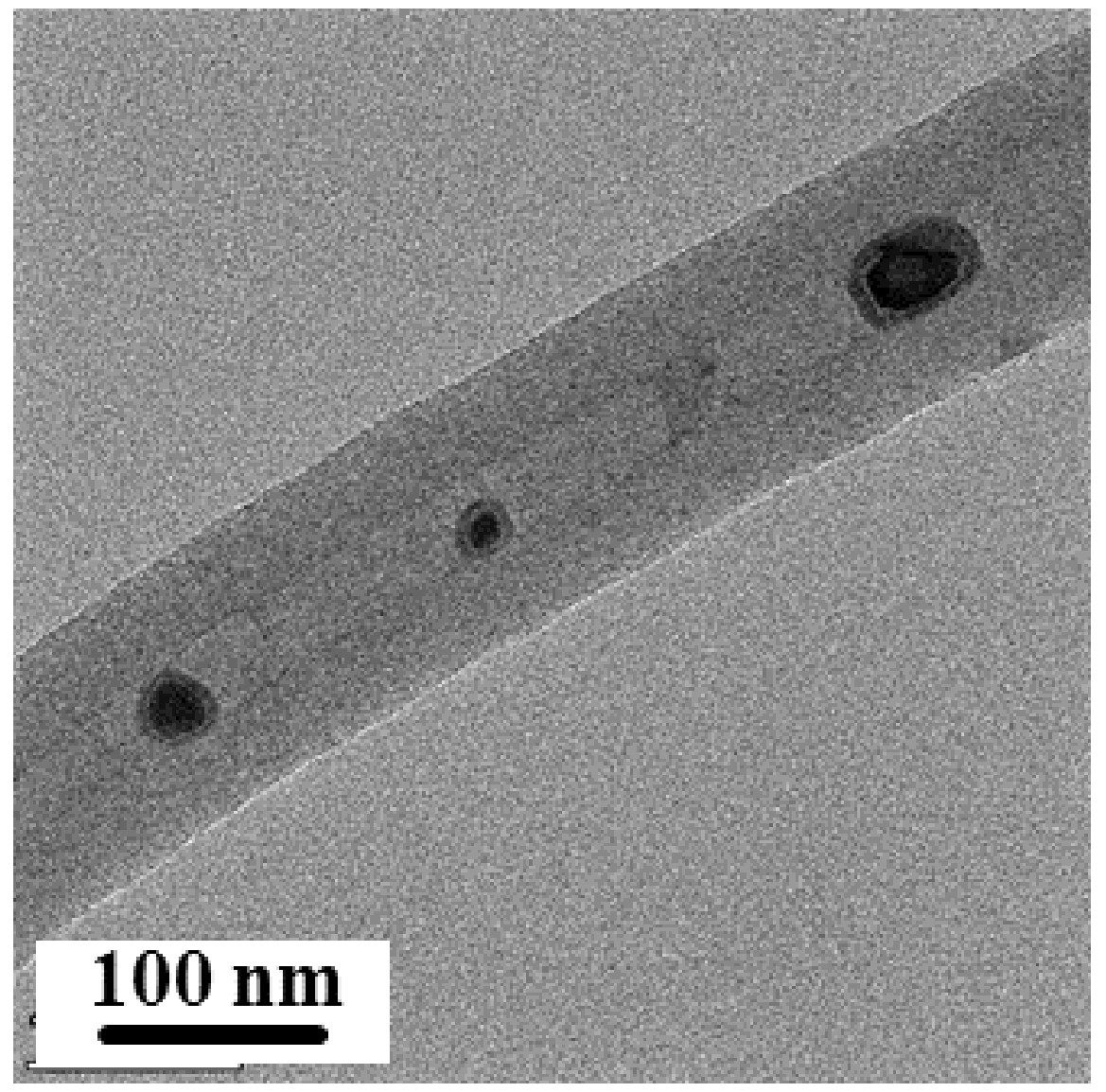




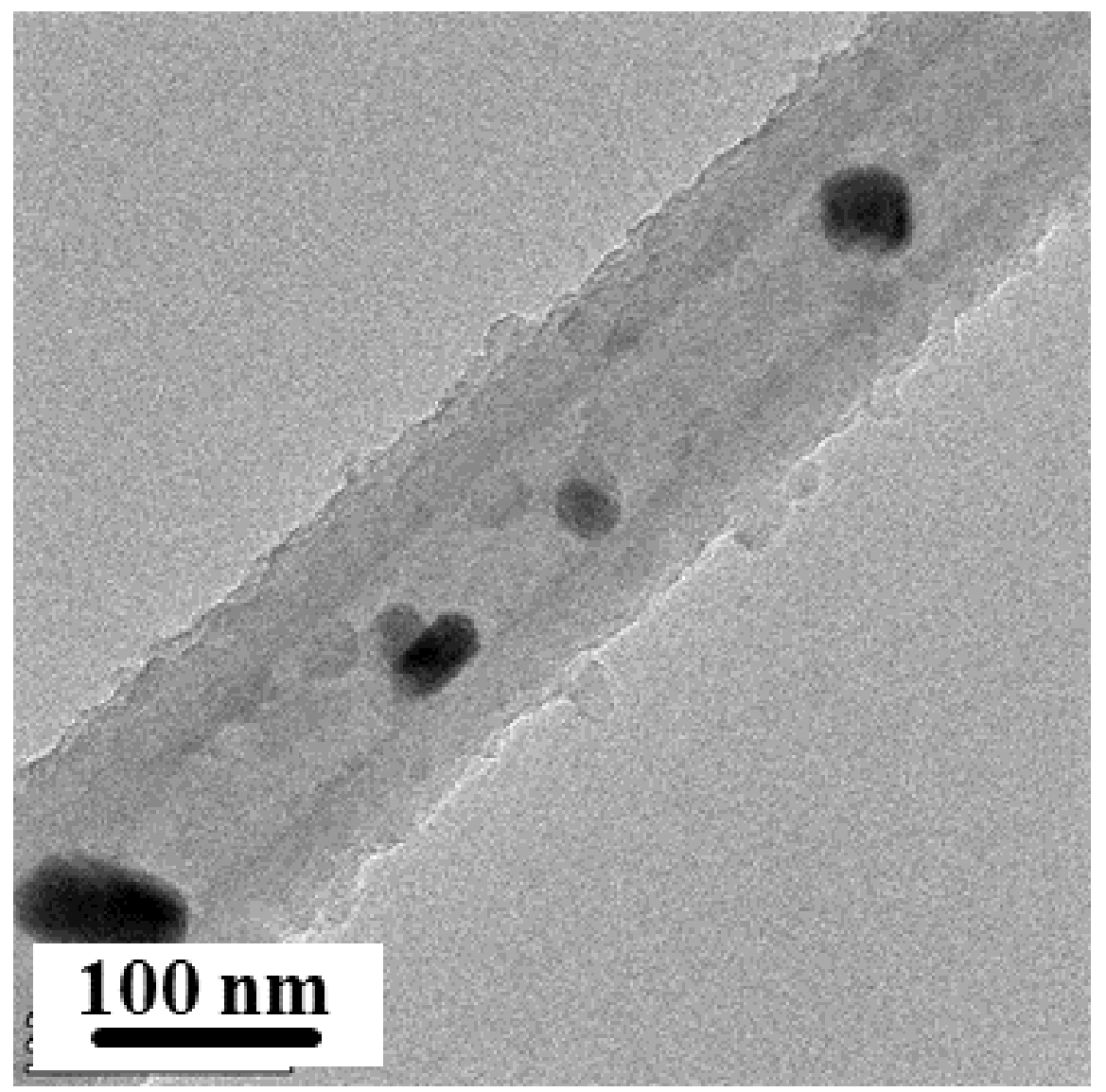




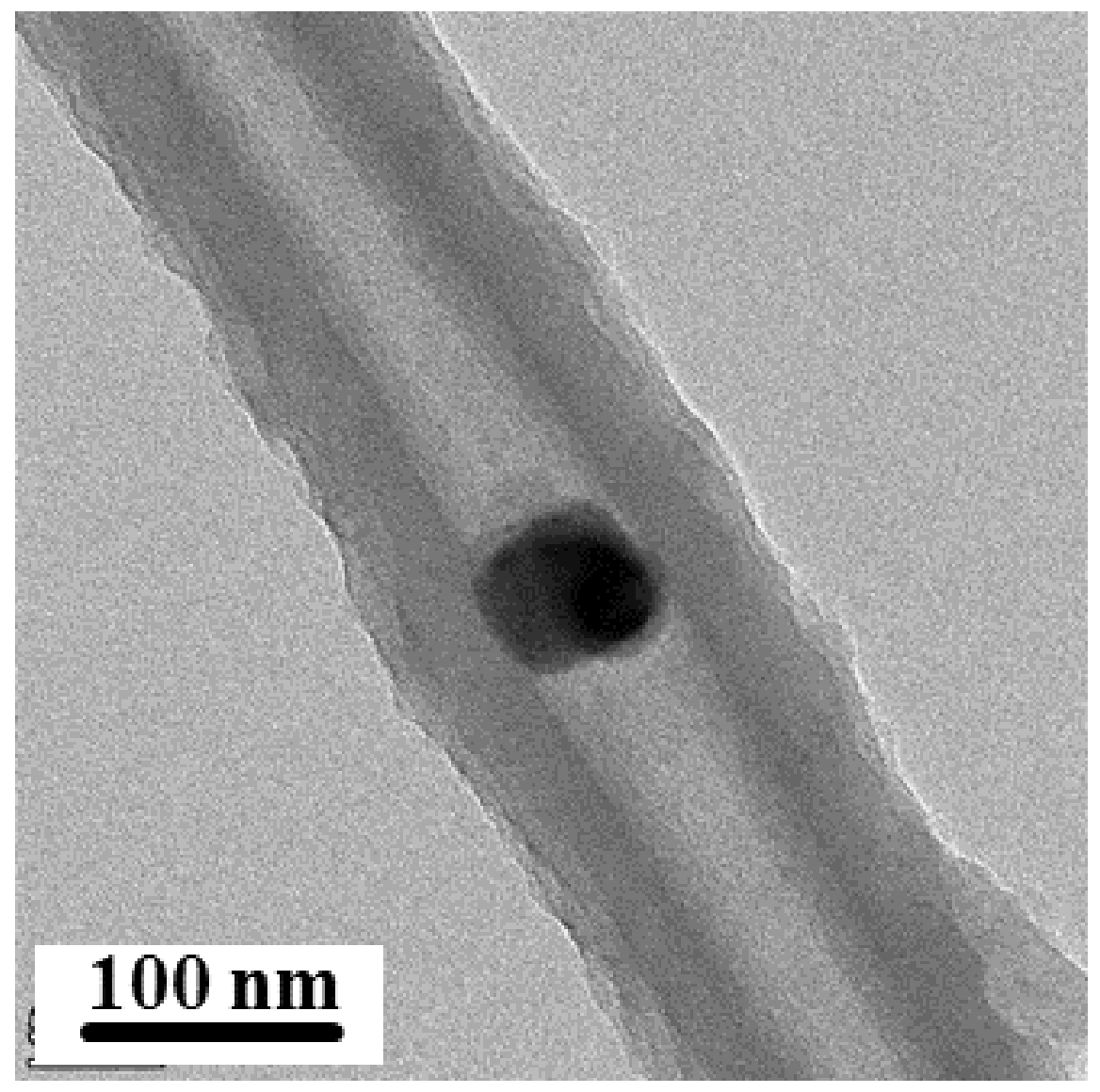




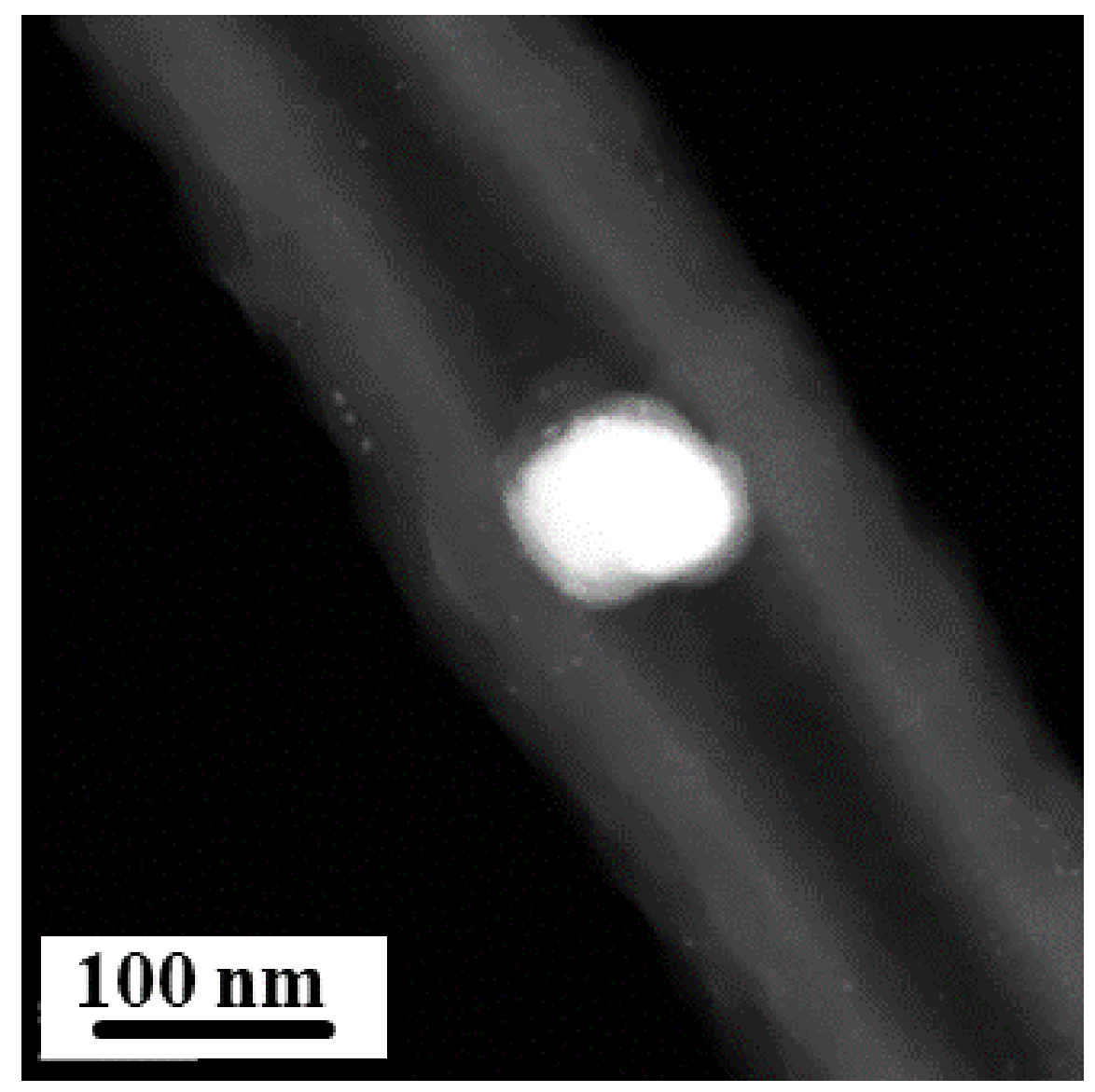




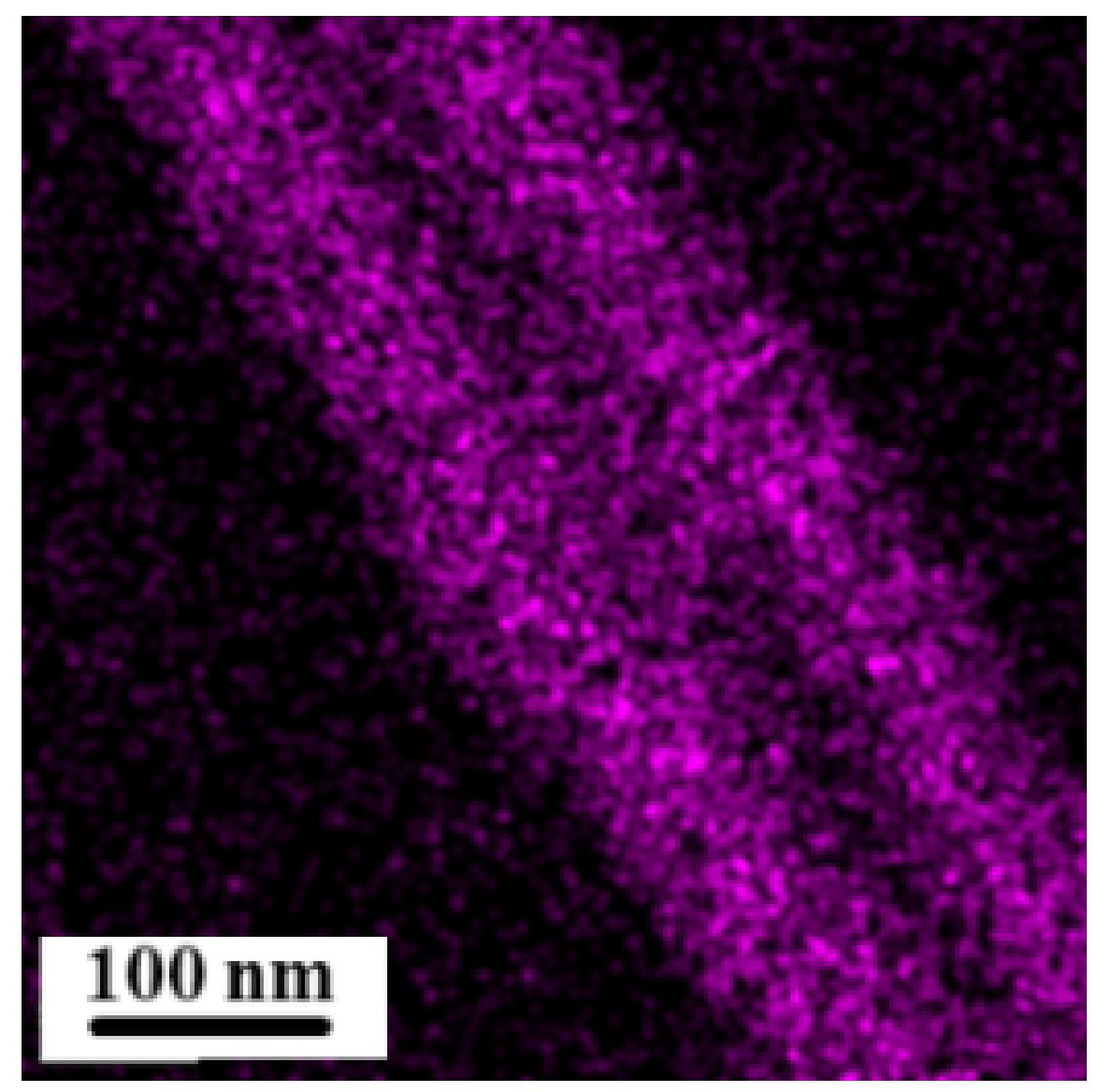




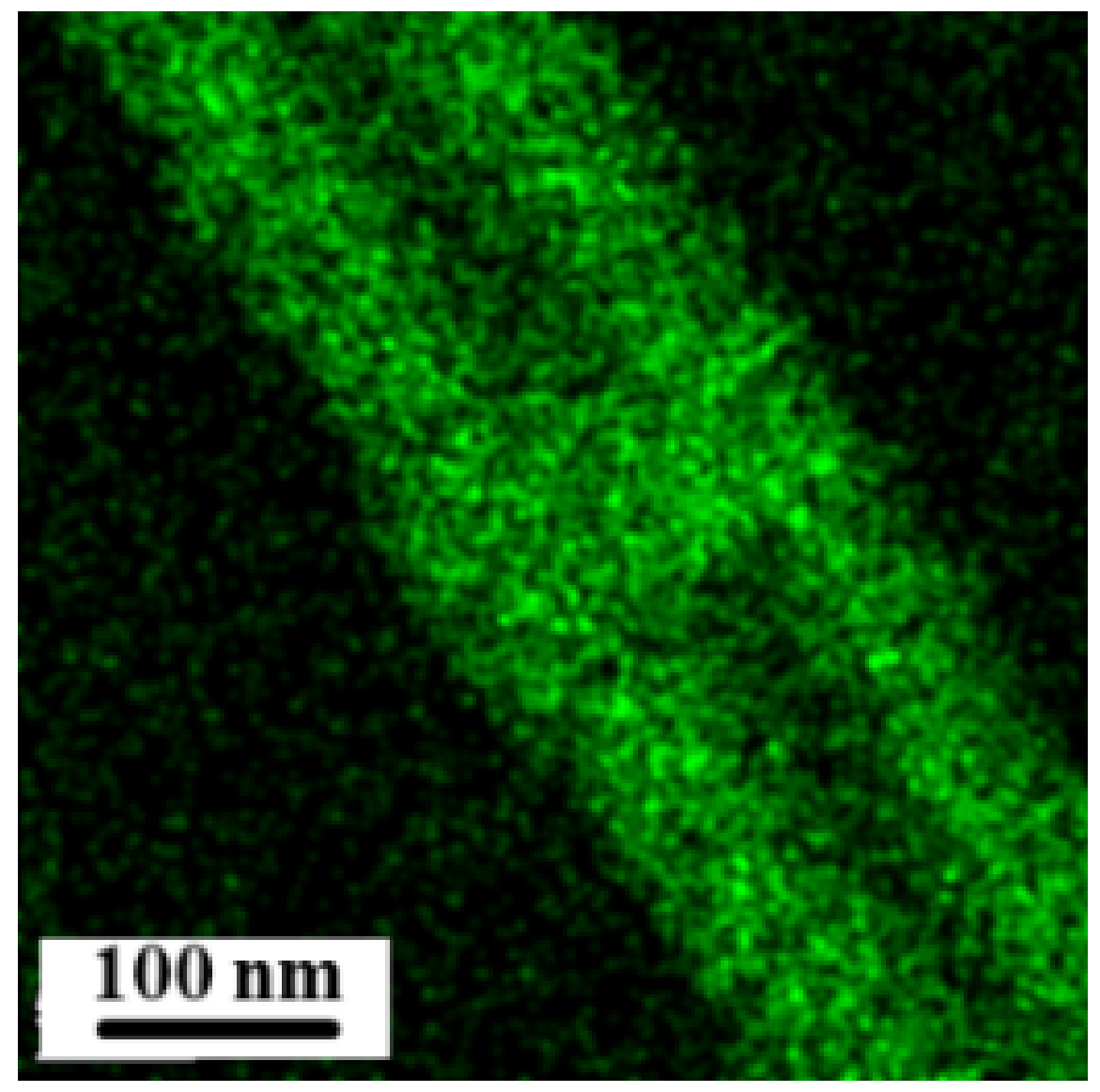




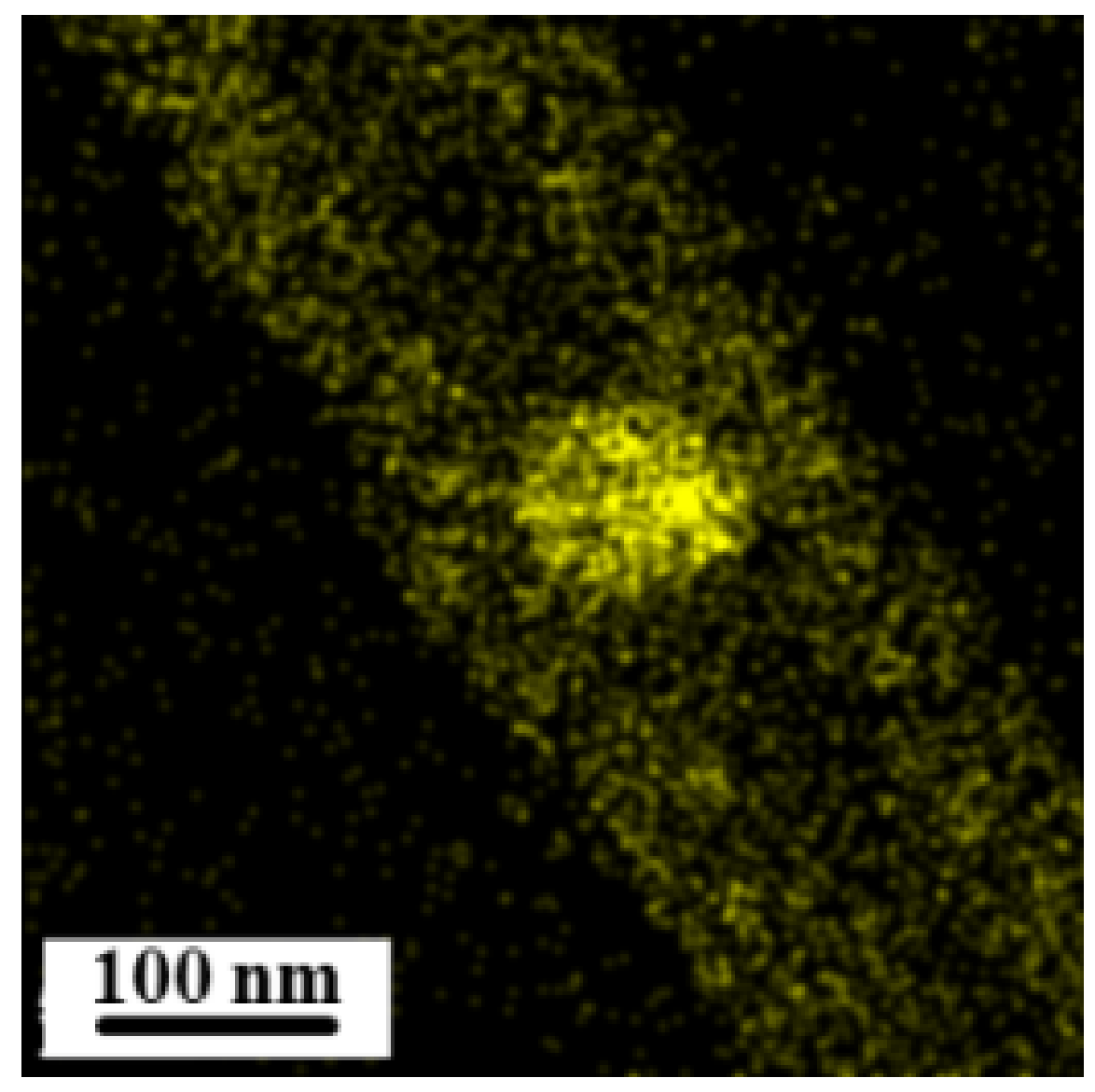




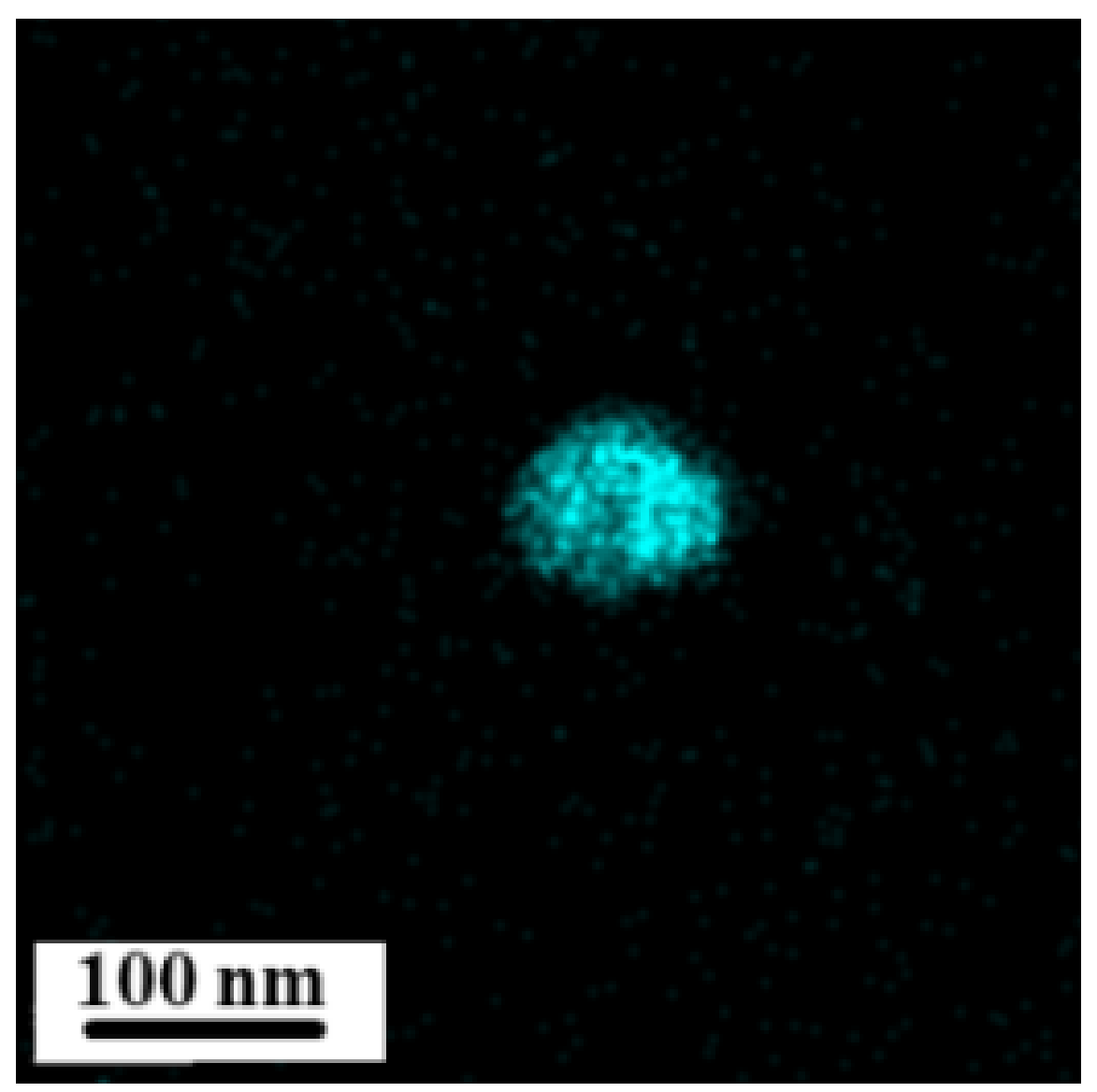

Figure 3 SEM (a-c) and TEM (e-g) images of MTCFs (a, e), BSA-MTCFs@SIP (b, f), MTCFs@SIP@CBMA (c, g); SEM images of MTCFs@NIP@CBMA (d); EDS analysis of MTCFs@SIP@CBMA: Full spectrum(h), $\mathrm{C}$ element (i), N element (g), O element (k), Fe(l)

Composition analysis

To confirm the composition of the polymer shell and prove the synthesis of MTCFs@SIP@CBMA, we systematically characterized the products at all steps. The FT-IR spectra of MTCFs, BSAMTCFs@SIP, BSA-MTCFs@SIP@CBMA, MTCFs@SIP@CBMA, and MTCFs@NIP@CBMA are shown in Figure 4a. Compared with the infrared curves of MTCFs, the absorption peaks of BSA-MTCFs@SIP, BSA-MTCFs@SIP@CBMA, MTCFs@SIP@CBMA, and MTCFs@NIP@CBMA at $1460 \mathrm{~cm}^{-1}$ were attributed to the aromatic ring on dopamine, which proved the successful coating of imprinted polymer. BSAMTCFs@SIP@CBMA, MTCFs@SIP@CBMA, and MTCFs@NIP@CBMA emerged absorption peaks at 1734 $\mathrm{cm}^{-1}$ attributable to -CO-O on CBMA, indicating that CBMA anti-protein adsorption segments were successfully grafted on the surface of BSA-MTCFs@SIP. Furthermore, the infrared spectra of BSA-MTCFs@SIP and BSA-MTCFs@SIP@CBMA both exhibited absorption peaks at $2568 \mathrm{~cm}^{-1}$. This band can be assigned to -SH on BSA, confirming the successful imprinting of BSA on the surface of fibers. After elution, the absorption peak of -SH in the infrared spectrum of MTCFs@SIP@CBMA disappeared. It was basically consistent with the absorption peak of MTCFs@NIP@CBMA, indicating that BSA was completely eluted.

The composition of organic and inorganic components in the nanofibers and the thermal stability of the nanofibers were analyzed by thermogravimetric analysis. The measured TGA curve is presented in Figure $4 \mathrm{~b}$. It was clear to see that all the samples had no obvious weight loss before 200, indicating superior thermal stability of nanofibers. As the temperature increased, MTCFs, BSA-MTCFs@SIP, and BSA- 
MTCFs@SIP@CBMA lost weight at different rates. MTCFs began to exhibit significant weight loss at 328 and was completely degraded at 369 . The weight loss rate was $86.18 \%$, which was caused by the combustion of carbon under oxygen. The degradation of BSA-MTCFs@SIP began at 268 and was complete at 387, with a weight loss rate of $89.11 \%$. The reason why BSA-MTCFs@SIP demonstrated lower pyrolysis temperature and higher weight loss rate than MTCFs was that the coating of PDA on its surface. Compared with BSAMTCFs@SIP, BSA-MTCFs@SIP@CBMA began to degrade at 237 and completely degraded at 390 . The weight loss rate was $91.39 \%$. The reincrease in weight loss rate confirmed the successful grafting of CBMA polymer segments on the surface of BSA-MTCFs@SIP.

The specific composition of the magnetic nanoparticles loaded in the tube of carbon nanofiber was analyzed by XRD. As shown in Figure 4c, three diffraction peaks appeared in MTCFs at diffraction angles $2 \vartheta$ of $44.67^{\circ}, 65.02^{\circ}$ and $82.33^{\circ}$, corresponding to (110), (200) and (211) crystal planes, respectively. According to the PDF card, the diffraction peak data was consistent with that of Fe (PDF 87-0721) with structure of body-centered cubic. Therefore, it could be inferred that the magnetic nanoparticles present in the carbon nanofiber tube were Fe nanoparticles. Additionally, the XRD diffraction peak positions of BSA-MTCFs@SIP and MTCFs@SIP@CBMA are basically consistent with that of MTFs. This meant that the coating process of PDA and the subsequent grafting of the CBMA anti-protein adsorption segments had no effect on the crystal form of Fe.

The magnetic properties of MTCFs, BSA-MTCFs@SIP, and MTCFs@SIP@CBMA were measured by VSM. The results are shown in Figure 4d. It can be seen that all the samples possessed magnetic properties. The specific saturation magnetization of MTFs was $8.29 \mathrm{emu} / \mathrm{g}$, while the specific saturation magnetization of BSA-MTCFs@SIP and MTCFs@SIP@CBMA were $7.25 \mathrm{emu} / \mathrm{g}$ and $6.21 \mathrm{emu} / \mathrm{g}$, respectively. The decrease of magnetic susceptibility was due to the fact that the magnetic responsiveness of the materials was weakened to some extent by both the coating of PDA and the grafting of CBMA polymer chains. Fortunately, this magnetic performance was sufficient to achieve rapid separation of the samples from the solution. Thus, the separation efficiency was improved.

Figure 4 FT-IR spectra of products at each stage (a); TGA curves of MTCFs, BSA-MTCFs@SIP and BSA-MTCFs@SIP@CBMA (b); XRD (c) and VSM (d) curves of MTCFs, BSA-MTCFs@SIP and MTCFs@SIP@CBMA

Characterization of specific surface area and pore performance

High specific surface area could increase the number of effective imprinted sites, thus improving the adsorption capacity. Therefore, nitrogen adsorption apparatus was applied to measure the specific surface area and pore properties of the materials. The nitrogen adsorption-desorption curves and pore size distribution curves of the samples are given in Figure 5. According to the nomenclature of the International Union of Pure and Applied Chemistry, the adsorption and desorption curves of MTCFs, BSA-MTCFs@SIP, BSA-MTCFs@SIP@CBMA, and MTCFs@SIP@CBMA all belonged to type IV. The hysteresis loop type was H4, indicating the slit-like pores in materials. It was worth noting that the specific surface area of the fibers decreased gradually as the procedure of PDA coating and CBMA polymer grafting progressed (see Table 1). This was because part of the pores of the fiber were blocked by the coating of the polymers. After elution, the specific surface area of MTCFs@SIP@CBMA increased compared with BSA-MTCFs@SIP@CBMA, confirming the formation of imprinted sites on the surface of fibers. From the pore size distribution curve, it was found that the pore size of fibers was mainly divided into two sections, $4-40 \mathrm{~nm}$ and $40-100 \mathrm{~nm}$. The pores in the range of 4-40 nm were attributed to the small pores on the tube wall of fibers and the imprinted cavities left by the eluted templates. This part of the pores allowed BSA molecules to diffuse into the inner cavity of the tube. The range of 40-100 $\mathrm{nm}$ corresponded to the diameter of the cavity in the tube. The large-scale cavity caused an osmotic pressure difference between the solution inside and outside the tube, which in turn gave MTCFs@SIP@CBMA selfdriven adsorption performance. The nitrogen adsorption-desorption curve type and pore size distribution of MTCFs@NIP@CBMA were consistent with those of MTCFs@SIP@CBMA (see Figure S1). Nevertheless, due to the absence of imprinting sites on its surface, the specific surface area $\left(170.53 \mathrm{~m}^{2} / \mathrm{g}\right)$ and pore volume $\left(0.02 \mathrm{~cm}^{3} / \mathrm{g}\right)$ of MTCFs@NIP@CBMA were lower than those of MTCFs@SIP@CBMA. 
Figure $5 \mathrm{~N}_{2}$ adsorption-desorption curves and pore size distribution curves of MTCFs (a, b), BSAMTCFs@SIP (c, d), BSA-MTCFs@SIP@CBMA (e, f) and MTCFs@SIP@CBMA (g, h)

Table 1 Specific surface area and pore performance data of samples

\begin{tabular}{llll}
\hline Samples & BET $\left(\mathrm{m}^{2} / \mathrm{g}\right)$ & Average pore size $(\mathrm{nm})$ & Pore volume $\left(\mathrm{cm}^{3} / \mathrm{g}\right)$ \\
\hline MTCFs & 339.9818 & 12.8218 & 0.085837 \\
BSA-MTCFs@SIP & 221.4250 & 17.9095 & 0.061691 \\
BSA-MTCFs@SIP@CBMA & 169.0844 & 28.4658 & 0.036203 \\
MTCFs@SIP@CBMA & 186.4907 & 25.9841 & 0.042046 \\
MTCFs@NIP@CBMA & 170.5277 & 25.1841 & 0.020789 \\
\hline
\end{tabular}

\subsection{Application performance of MTCFs@SIP@CBMA}

\subsubsection{Adsorption kinetics}

Under the premise that the initial concentration of BSA was $0.1 \mathrm{mg} / \mathrm{mL}$, the relationship between the adsorption capacity of MTCFs@SIP@CBMA and MTCFs@NIP@CBMA on BSA over time was measured. As shown in Figure 6, the adsorption process was obviously divided into four stages. At 0-30 min, the adsorption capacity of MTCFs@SIP@CBMA on BSA gradually increased with time. The reason was described as that BSA first bound to the imprinted sites on the surface of the fibers relying on electrostatic attraction, hydrogen bonding and hydrophobic interaction. As time gone on, the binding amount increased. At 30-60 min, the adsorption capacity remained unchanged, indicating that the imprinted sites on MTCFs@SIP@CBMA surface reached saturation. The saturated adsorption capacity for BSA was $395.26 \mathrm{mg} / \mathrm{g}$. However, the osmotic pressure difference between the inside and outside of the tube would drive BSA to migrate into the cavity through the pores on the tube wall. The balance of adsorption capacity suggested that the adsorbed BSA still occupied the imprinting sites. At 30-100 min, the adsorption capacity increased again with time, and the adsorption capacity was significantly higher than the equilibrium adsorption capacity. This illustrated that the BSA on the surface of MTCFs@SIP@CBMA disengaged from the imprinting sites and diffused into the cavity. At this time, the remaining BSA in the solution again adsorbed to the imprinting sites on the surface. After $100 \mathrm{~min}$, the BSA migrating into the cavity migrated out of the fiber port and migrated outward at a rate greater than the adsorption rate, so the adsorption amount decreased with time. The above adsorption results well confirmed the self-driven adsorption performance of MTCFs@SIP@CBMA. For MTCFs@NIP@CBMA, owing to the lack of imprinting sites matching with BSA on its surface and the grafting of anti-protein adsorption chain segments, the adsorption capacity was significantly lower than MTCFs@SIP@CBMA (only 57.42 mg/g). Simultaneously, because MTCFs@NIP@CBMA had no imprinted cavity connected to the carrier fibers and the coated PDA and CBMA polymers were compact, the adsorption process of MTCFs@NIP@CBMA did not exhibit self-driven adsorption performance.

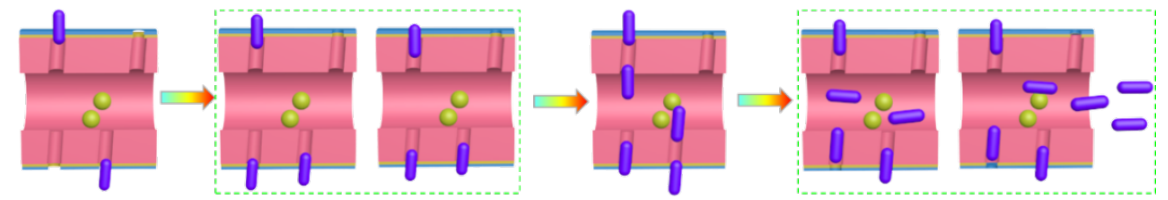

Figure 6 The adsorption kinetics of BSA on MTCFs@SIP@CBMA and MTCFs@NIP@CBMA. Error bars represent \pm standard deviations, $\mathrm{n}=3$.

\subsubsection{Adsorption thermodynamics}

The adsorption capacities of MTCFs@SIP@CBMA and MTCFs@NIP@CBMA varied with the initial concentration of BSA as shown in Figure 7. For MTCFs@SIP@CBMA, when the concentration of BSA was lower 
than $0.1 \mathrm{mg} / \mathrm{mL}$, the adsorption capacity gradually increased with the increase of concentration. When the concentration reached $0.1 \mathrm{mg} / \mathrm{mL}$, the adsorption capacity tended to be stable. This was an indication that the adsorption capacity of MTCFs@SIP@CBMA on BSA achieved saturation at this moment. The equilibrium adsorption capacity was $392.75 \mathrm{mg} / \mathrm{g}$, which was significantly higher than that of MTCFs@NIP@CBMA at $57.96 \mathrm{mg} / \mathrm{g}$. Consequently, the calculated imprinting factor was 6.78 . The outcomes illustrated that the recombination of BSA on MTCFs@SIP@CBMA occurred based on the imprinting sites. Otherwise, the imprinting effect was significantly improved due to the introduction of anti-protein adsorption chain CBMA, which reduced the non-specific adsorption. Compared with the reported adsorption properties and IF values of imprinted materials (see Table 2), MTCFs@SIP@CBMA exhibited fast adsorption rate, high adsorption capacity and considerable IF values, which could achieve efficient protein separation in solution.

Figure 7 Adsorption isotherms of MTCFs@SIP@CBMA and MTCFs@NIP@CBMA for BSA. Error bars represent \pm standard deviations, $\mathrm{n}=3$.

Table 2 Comparison of $\mathrm{Q}_{\mathrm{MIP}}$, adsorption equilibrium time and IF for reported imprinted materials

\begin{tabular}{|c|c|c|c|c|c|c|}
\hline $\begin{array}{l}\text { Protein } \\
\text { imprinted } \\
\text { materials }\end{array}$ & Carrier & $\begin{array}{l}\text { Template } \\
\text { protein }\end{array}$ & $\mathrm{Q}_{\mathrm{MIP}}(\mathrm{mg} / \mathrm{g})$ & Time $(\min )$ & IF & Ref. \\
\hline \multicolumn{3}{|c|}{ 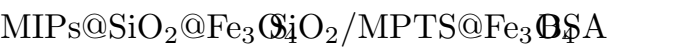 } & 86.87 & 60 & 6.49 & 10 \\
\hline \multicolumn{3}{|c|}{$\mathrm{Fe}_{3} \mathrm{O}_{4} @ \mathrm{MnO}_{2} @ \mathrm{MFE}_{3} \mathrm{O}_{4} @ \mathrm{MnO}_{2} \quad$ BSA } & 140.66 & 90 & 3.3 & 38 \\
\hline \multicolumn{3}{|c|}{ MWMS@MIPs MWMS } & 146.2 & 60 & 4.36 & 42 \\
\hline \multicolumn{3}{|c|}{$\begin{array}{ll}\mathrm{Fe}_{3} \mathrm{O}_{4} @ \mathrm{IL} @ \mathrm{MIP} & \mathrm{Fe}_{3} \mathrm{O}_{4} \\
\mathrm{MSS} @ \mathrm{Ni}^{2+} & \mathrm{Fe}_{2} \mathrm{O}_{4} @ \mathrm{mSiO}_{2}\end{array}$} & 50.6 & 60 & 3.33 & 43 \\
\hline \multicolumn{7}{|l|}{ MIPs } \\
\hline PMMIPs & MHNTs & BSA & 258.4 & 80 & 2.44 & 45 \\
\hline \multicolumn{2}{|c|}{ MTCFs@SIP@CBIMIACFs } & BSA & 395.26 & 30 & 6.88 & This work \\
\hline
\end{tabular}

*MWMS: magnetic composite microspheres with a wrinkled surface; MHNTs: magnetic halloysite nanotubes; MPTS: $\gamma$-methacryloxypropyltrimethoxysilane

The adsorption isotherm data of MTCFs@SIP@CBMA and MTCFs@NIP@CBMA to BSA were further evaluated using Langmuir and Freundlich isotherm models. The fitted linear curve is shown in Figure S2, and relevant parameters are listed in Table 3. By comparing $\mathrm{R}^{2}$, it can be seen that the Langmuir model could better fit the adsorption isotherm data of MTCFs@SIP@CBMA and MTCFs@NIP@CBMA. This illustrated that the adsorption process was monolayer adsorption. The theoretical maximum adsorption capacities of MTCFs@SIP@CBMA and MTCFs@NIP@CBMA calculated by Langmuir model were 421.69 $\mathrm{mg} / \mathrm{g}$ and $69.37 \mathrm{mg} / \mathrm{g}$. These were basically consistent with the experimental data.

Table 3 Related parameters of Langmuir and Freundlich isotherm models obtained by fitting

\begin{tabular}{lllllll}
\hline Samples & Langmuir & Langmuir & Langmuir & Freundlich & Freundlich & Freundlich \\
\hline & $\mathrm{q}_{\mathrm{m}}\left(\mathrm{mg} \cdot \mathrm{g}^{-1}\right)$ & $\mathrm{K}_{\mathrm{L}}\left(\mathrm{L} \cdot \mathrm{mg}^{-1}\right)$ & $\mathrm{R}^{2}$ & $\mathrm{~K}_{\mathrm{F}}\left(\mathrm{mg}^{1-\mathrm{n}} \cdot \mathrm{L}^{\mathrm{n}} \cdot \mathrm{g}^{-1}\right)$ & $1 / \mathrm{n}$ & $\mathrm{R}^{2}$ \\
MTCFs@SIP@CBMA & 421.69 & 30.46 & 0.9831 & 1000.64 & 0.44 & 0.9695 \\
MTCFs@NIP@CBMA & 69.37 & 22.59 & 0.9796 & 180.98 & 0.51 & 0.9711 \\
\hline
\end{tabular}

\subsubsection{Recognition selectivity of MTCFs@SIP@CBMA}

Taking isoelectric point and molecular weight as investigating factors, OVA, HSA, Lyz, and Bhb were selected as protein analogues to investigate the recognition selectivity of MTCFs@SIP@CBMA to BSA. The adsorption capacities of MTCFs@SIP@CBMA and MTCFs@NIP@CBMA on BSA and other four proteins 
are illustrated in Figure 8. The IF values are listed above the histogram. Obviously, MTCFs@SIP@CBMA exhibited a higher adsorption capacity and IF for BSA than the other four proteins. This was explained by that BSA matched the imprinting sites of MTCFs@SIP@CBMA in three-dimensional space and functional group arrangement. HSA not only possessed an isoelectric point and molecular weight similar to BSA, but also their three-dimensional conformation and $60 \%$ amino acid sequence were also very similar. Thus, the adsorption capacity and IF of MTCFs@SIP@CBMA to HSA ranked second. However, the adsorption capacity and IF of HSA were much lower than those of BSA because the exposed functional groups were different from BSA and were affected by the anti-protein adsorption segments. The isoelectric point of OVA was similar to that of BSA, but the molecular weight was smaller. The molecular weight of Bhb was close to that of BSA, while Lyz had a smaller molecular weight. However, due to the mismatching of charge and the effect of anti-protein adsorption chain segments, the adsorption capacities and IF values of MTCFs@SIP@CBMA for Bhb and Lyz were not high. In view of above analysis, the order of adsorption capacities and IF values of MTCFs@SIP@CBMA for the other four proteins was HSA > OVA > Bhb > Lyz. The $\beta$ values calculated by IF are listed in Table S1. The $\beta$ values of MTCFs@SIP@CBMA for OVA, HSA, Lyz and Bhb were 5.81, 5.25, 6.67 and 6.03, indicating that MTCFs@SIP@CBMA could selectively identify BSA. From above results the conclusion can be inferred that the introduction of CBMA anti-protein adsorption chain segments can indeed improve the recognition selectivity of imprinted materials.

Figure 8 Selective adsorption of MTCFs@SIP@CBMA and MTCFs@NIP@CBMA toward BSA, OVA, HSA, Lyz and Bhb. Error bars represent \pm standard deviations, $\mathrm{n}=3$.

\subsubsection{Competitive adsorption}

In order to further evaluate the recognition selectivity of MTCFs@SIP@CBMA fibers, adsorption experiments were carried out with binary mixed protein of Bhb and BSA as adsorption objects. The mixed proteins $\left(\mathrm{C}_{\mathrm{Bhb}}=\mathrm{C}_{\mathrm{BSA}}=0.1 \mathrm{mg} / \mathrm{mL}\right)$ were adsorbed with $1 \mathrm{mg}$ MTCFs@SIP@CBMA or MTCFs@NIP@CBMA for 30 min. After magnet separation, the absorption spectrum of proteins in supernatants was scanned by UV-vis spectrophotometer with a scanning range of 250-550 nm. As can be seen from Figure 9a, the absorbance of the mixed proteins at $278 \mathrm{~nm}$ decreased significantly after adsorption by MTCFs@SIP@CBMA, while the absorbance at $409 \mathrm{~nm}$ remained almost unchanged. When MTCFs@NIP@CBMA was selected as the adsorbent, BSA was adsorbed and Bhb was simultaneously adsorbed (the absorbance at $278 \mathrm{~nm}$ and 409 nm were both reduced). The results showed that MTCFs@SIP@CBMA could selectively recognize BSA in binary mixed proteins while MTCFs@NIP@CBMA could not. Moreover, SDS-PAGE gel electrophoresis was used to analyze whether MTCFs@SIP@CBMA can specifically recognize BSA from the five-component mixed protein solutions of BSA, OVA, HSA, Lyz, and Bhb. The results are given in Figure 9b. Compared with lane 2, the intensity of the corresponding BSA band in channel 3 was weakened, while the intensity of other protein bands hardly changed. Compared lane 4 with lane 2, it was found that the band strength of each protein did not change significantly. In lane 5, only a BSA band was present in the primary eluent fractions of MTCFs@SIP@CBMA. The above results indicated that MTCFs@SIP@CBMA could also specifically recognize BSA from multicomponent mixed proteins. In summary, MTCFs@SIP@CBMA demonstrated outstanding recognition selectivity for BSA, which was ascribed to the reduction of non-specific adsorption by anti-protein adsorption chain CBMA grafted on the surface of the imprinted layer. 


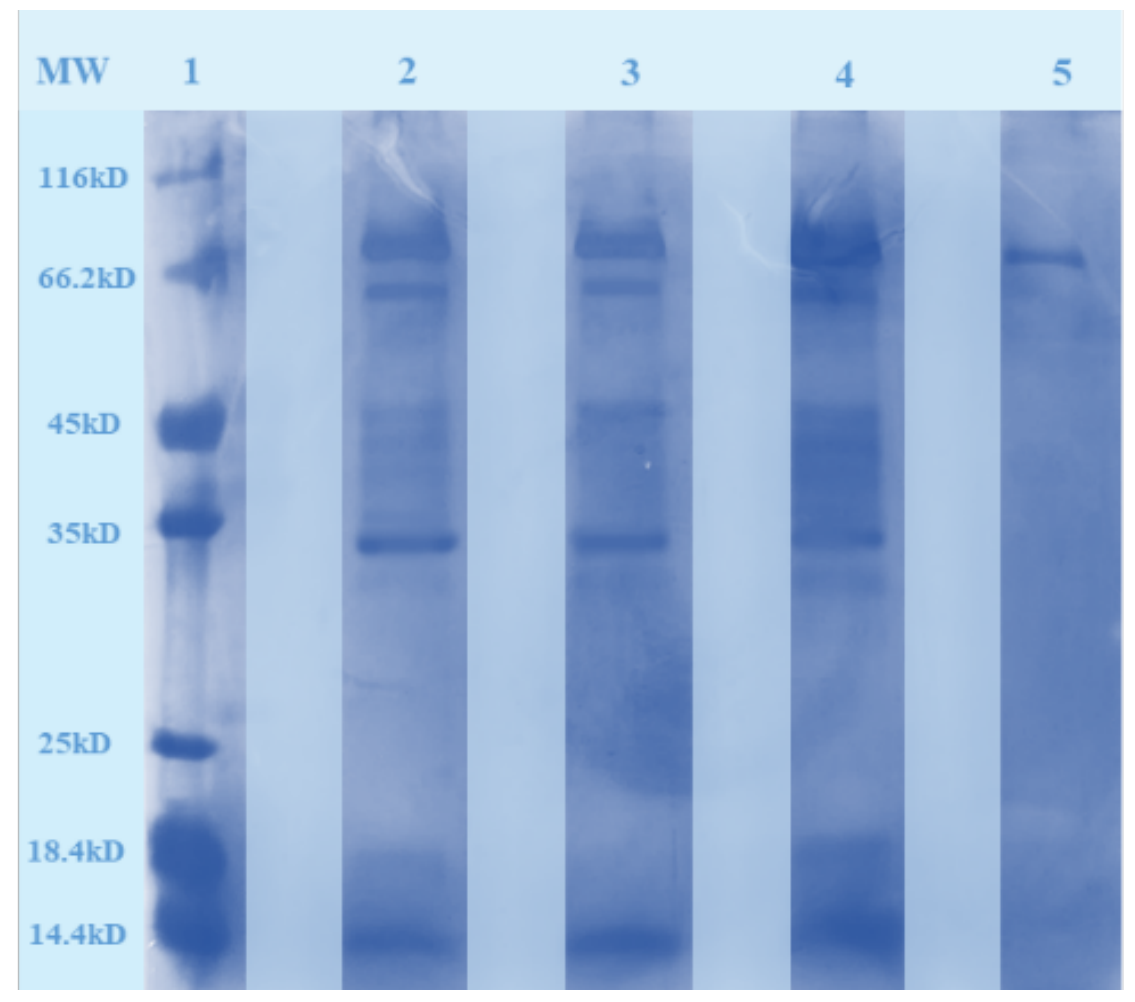

Figure 9 UV spectra of binary solution of BSA-Bhb on MTCFs@SIP@CBMA and MTCFs@NIP@CBMA (a); SDS-PAGE analysis of adsorption for BSA by MTCFs@SIP@CBMA from protein mixture (b). Lane 1: marker; lane 2: protein mixed solution; lane 3: remaining protein mixture after adsorption by MTCFs@SIP@CBMA; lane 4: remaining protein mixture after adsorption by MTCFs@NIP@CBMA; lane 5: the eluent from MTCFs@SIP@CBMA; The dosage of protein solution was $10 \mu \mathrm{L}$.

Real sample analysis

Selecting fetal bovine serum (FBS) as a complex biological sample, the ability of MTCFs@SIP@CBMA to selectively isolate BSA from real samples was investigated. The results of UV spectra and SDS-PAGE gel electrophoresis analysis of the 30-fold diluted FBS stock solution, protein supernatants after adsorption by MTCFs@SIP@CBMA and MTCFs@NIP@CBMA, and the primary eluent of MTCFs@SIP@CBMA are shown in Figure 10. As observed from Figure 10a, the absorbance of FBS at $278 \mathrm{~nm}$ was significantly reduced after adsorption by MTCFs@SIP@CBMA. At the same time, the eluate of MTCFs@SIP@CBMA only appeared an absorption peak at $278 \mathrm{~nm}$ attributable to BSA, indicating that MTCFs@SIP@CBMA could specifically bind BSA from FBS. Furthermore, only a BSA band appeared in lane 5 of the corresponding electrophoretic photo (Figure. 10b), confirming again that MTCFs@SIP@CBMA can selectively isolate and capture BSA from real FBS. As a result, MTCFs@SIP@CBMA was expected to achieve the separation and enrichment of BSA in complex biological samples. 


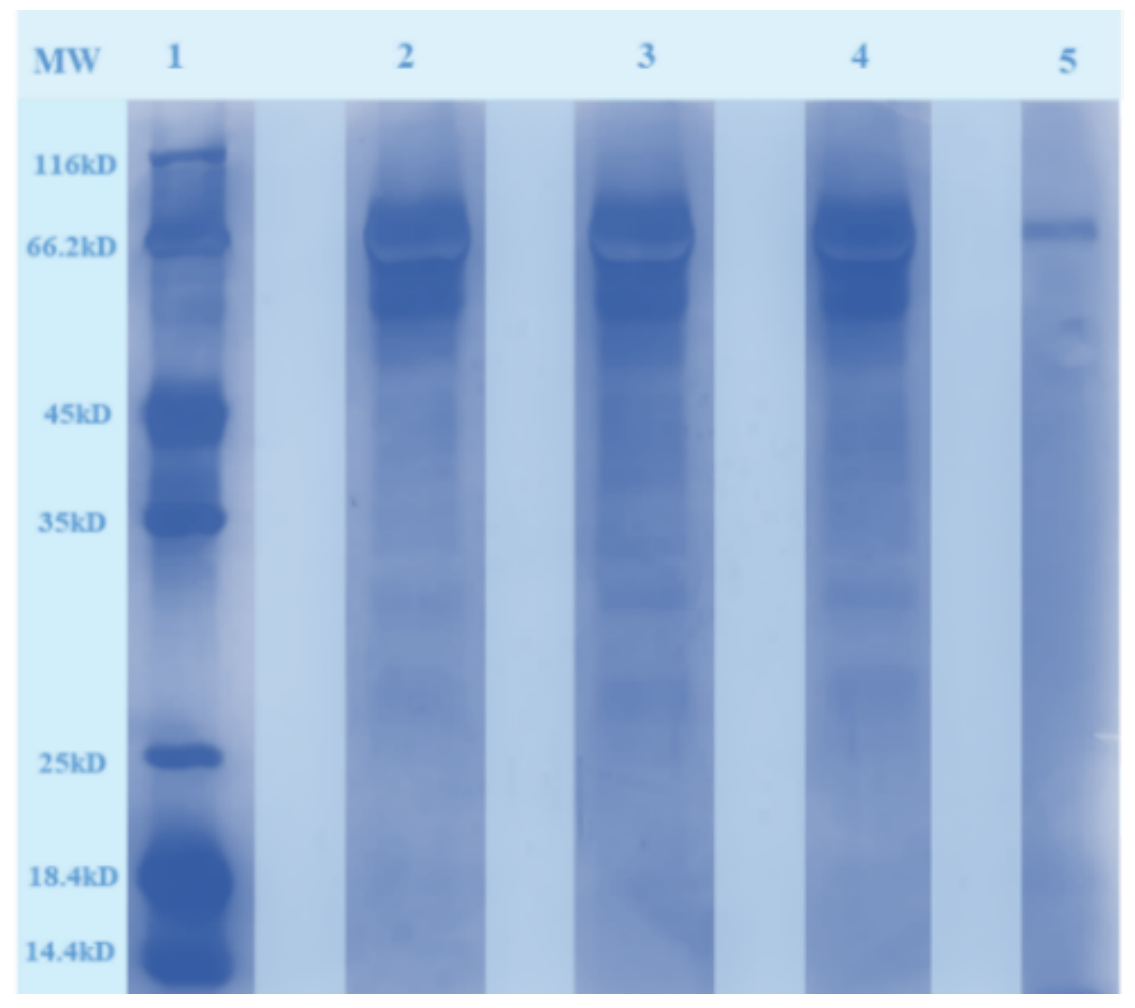

Figure 10 UV spectrum of FBS before and after adsorption by MTCFs@SIP@CBMA and MTCFs@NIP@CBMA (a); SDS-PAGE analysis of adsorption for BSA by MTCFs@SIP@CBMA from FBS (b). Lane 1: marker; lane 2: FBS stock solution; lane 3: remaining FBS solution after adsorption by MTCFs@SIP@CBMA; lane 4: remaining FBS solution after adsorption by MTCFs@NIP@CBMA; lane 5: the eluent from MTCFs@SIP@CBMA; The dosage of protein solution was $10 \mu \mathrm{L}$.

\section{Reusability of MTCFs@SIP@CBMA}

Reusability played a very important role for the popularization and application of imprinted materials. In this section, after adsorbing BSA with the same MTCFs@SIP@CBMA and MTCFs@NIP@CBMA, the adsorbed template BSA was eluted with $6 \%$ HAc. The adsorption-desorption process was repeated eight times and the adsorption amount was measured each time. As shown in Figure 11a, the adsorption capacity of MTCFs@SIP@CBMA on BSA only lost $4.76 \%$ after eight cycles. This loss was caused by the incomplete template elution and the destruction of the imprinting sites due to repeated elution processes. Compared with MTCFs@SIP@CBMA, due to the absence of imprinting sites on MTCFs@NIP@CBMA, the effect of the elution procedure on its adsorption capacity was negligible. Moreover, the morphology of MTCFs@SIP@CBMA after eight cycles of recycling was observed. The SEM and TEM photos are shown in Figure 11b and c. It can be seen that MTCFs@SIP@CBMA maintained a good hollow tubular structure. The VSM curve of MTCFs@SIP@CBMA after eight repeated cycles is shown in Figure 11d. It was observed that the repeated elution process hardly had effect on the magnetic properties of MTCFs@SIP@CBMA. The specific saturation magnetization after eight uses was $6.11 \mathrm{emu} / \mathrm{g}$. Based on the above analysis results, MTCFs@SIP@CBMA possessed excellent reusability, which provided a possibility for further application expansion. 

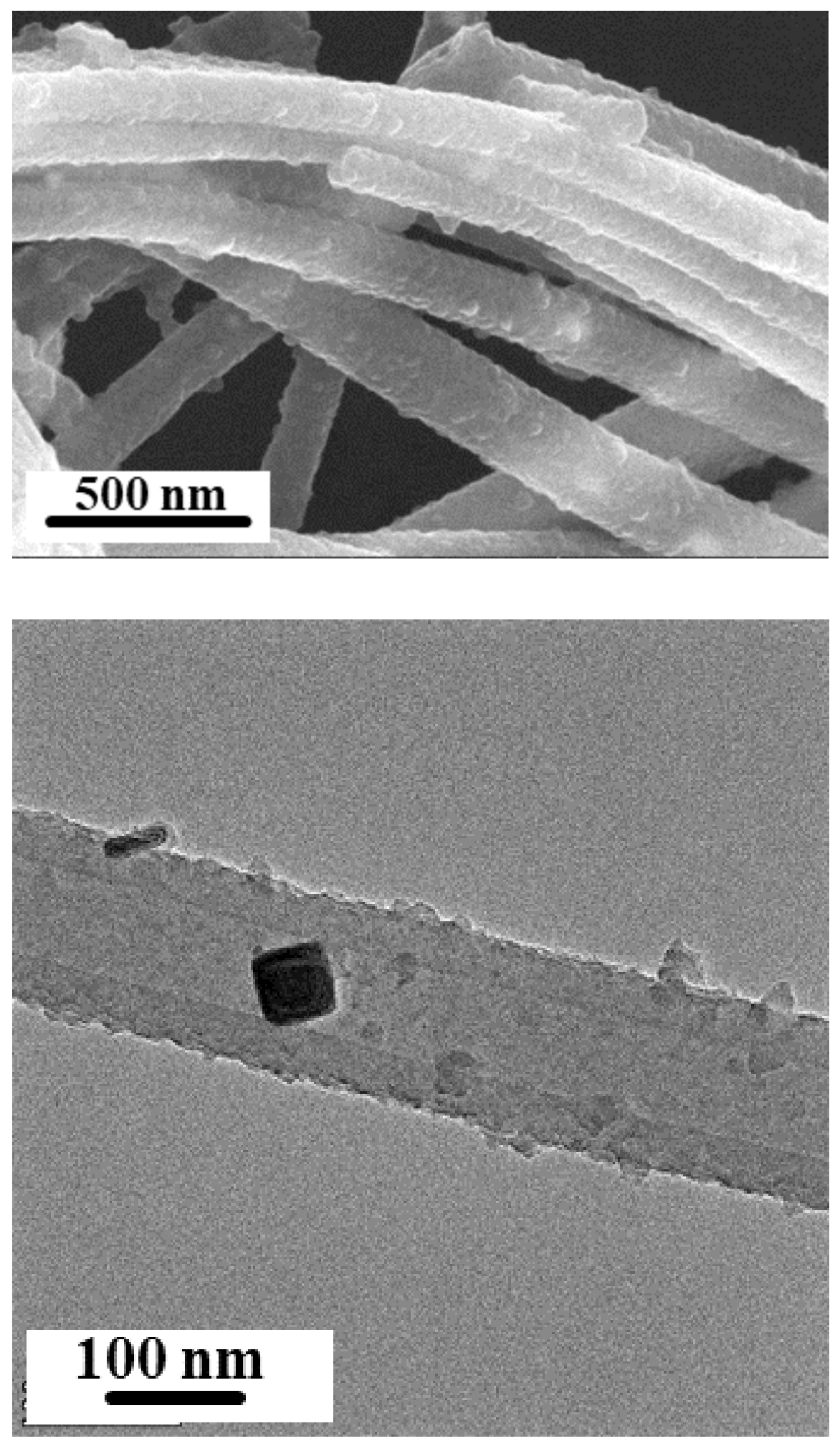

Figure 11. Reusability of MTCFs@SIP@CBMA and MTCFs@NIP@CBMA (a), SEM (b), TEM images (c), and VSM curves (d) of MTCFs@SIP@CBMA after 8 cycles. Error bars represent \pm standard deviations, $\mathrm{n}$ 
$=3$.

\section{Conclusion}

Dedicated to the construction of high-performance imprinted materials, this work developed an anti-protein adsorption segment CBMA-assisted self-driven BSA surface imprinted magnetic tubular carbon nanofibers MTCFs@SIP@CBMA with high recognition selectivity using a strategy of combining magnetic nanomaterial surface imprinting technique with amine-Michael addition. The carrier is magnetic tubular carbon nanofibers (MTCFs) prepared by hypercrosslinking polymerization and vacuum high-temperature carbonization. The special structure of MTCFs including porous tube wall, the cavity in the tube and the iron nanoparticles loaded in the tube endows MTCFs@SIP@CBMA with magnetic performance and self-driven adsorption performance, which simplifies the separation process while improving the adsorption capacity and speeding up the adsorption rate. The adsorption kinetics study confirms that MTCFs@SIP@CBMA exhibits significant self-driven adsorption characteristics. The saturated adsorption capacity is $395.26 \mathrm{mg} / \mathrm{g}$ with an imprinting factor of 6.88. To improve the recognition selectivity of imprinted materials on the premise of ensuring high adsorption capacity, biofriendly dopamine is selected as a functional monomer for the coating of imprinted layer. At the same time, the imprinted surface is grafted with CBMA polymer chain by the method of amino-Michael addition to reduce non-specific adsorption. This method makes the length and position of the CBMA polymer chain controllable. When $\mathrm{W}_{\mathrm{BSA}-\mathrm{MTCF} @ \mathrm{SIP}} / \mathrm{W}_{\mathrm{CBMA}}$ was 1:10, the length of CBMA polymer chain is the most suitable. Correspondingly, MTCFs@SIP@CBMA possesses the strongest antinonspecific adsorption effect, which in turn demonstrates the highest imprinting factor for BSA. Due to the contribution of anti-protein adsorption chain segments, MTCFs@SIP@CBMA presents excellent ability to specifically recognize BSA in both competitive adsorption and actual sample separation experiments. Furthermore, the adsorption capacity of MTCFs@SIP@CBMA lose only 4.76\% after eight times of repeated adsorptions-desorption, indicating its excellent reusability. All in all, the high-performance protein imprinted material MTCFs@SIP@CBMA provides potential practical application value and is expected to play a role in the development of protein drugs and drug diagnosis.

\section{Acknowledgments}

The authors are grateful for the support provided by the National Natural Science Foundation of China (No. 21975206, 52063029).

\section{References}

1. Adam H, Gopinath S C B, Arshad M K M., Adam T, Hashim U. Perspectives of nanobiotechnology and biomacromolecules in parkinson's disease.Process Biochem 2019; 86: 32-39.

2. Yang Z, Cui Y, Zhang Y, Liu P, Zhang Q, Zhang B. Identification of imprinted sites by fluorescence detection method based on reversible dynamic bond modified template protein. Compos. B. Eng. 2021; 223: 109154.

3. Zhao M, Huang S, Xie H, Wang J, Zhao X, Li M, Zhao M. Construction of Specific and Reversible Nanoreceptors for Proteins via Sequential Surface-Imprinting Strategy. Anal Chem 2020; 92: 10540-10547.

4. Guoning C, Pengqi G, Yan W, Lu W, Hua S, Yunzhe L, Wanghui J, Chun C, Qiang F. Preparation of molecularly imprinted polymers and application in a biomimetic biotin-avidin-ELISA for the detection of bovine serum albumin. Talanta 2019; 198: 55-62.

5. Zhao W, Li B, Xu S, Zhu Y, Liu X. A fabrication strategy for protein sensors based on an electroactive molecularly imprinted polymer: Cases of bovine serum albumin and trypsin sensing. Anal Chim Acta 2020; 1117: 25-34.

6. Wang Y, Zhou J, Wu C, Tian L, Zhang B, Zhang Q. Fabrication of micron-sized BSA-imprinted polymers with outstanding adsorption capacity based on poly(glycidyl methacrylate)/polystyrene (PGMA/PS) anisotropic microspheres. J Mater Chem B 2018; 6: 5860-5866. 
7. Yang C, Ji X F, Cao W Q, Wang J, Zhang Q, Zhong T L, Wang Y. Molecularly imprinted polymer based sensor directly responsive to attomole bovine serum albumin. Talanta 2019; 196: 402-407.

8. Yuan Y, Yang C, Lv T, Qiao F, Zhou Y, Yan H. Green synthesis of hydrophilic protein-imprinted resin with specific recognition of bovine serum albumin in aqueous matrix. Anal Chim Acta 2018; 1033: 213-220.

9. Zhai Y Y, Yun Y B, Li C L. Preparation of bovine serum albumin molecularly imprinted polymer by precipitation polymerization. J Dispers Sci Technol 2019; 41: 1371-1380.

10. Wang Y, Ma Y, Zhou J, Su K, Zhang B, Zhang, Q. Thermo-sensitive surface molecularly imprinted magnetic microspheres based on bio-macromolecules and their specific recognition of bovine serum albumin. J Sep Sci 2020; 43: 996-1002.

11. Fan J-P, Yu J-X, Yang X-M, Zhang X-H, Yuan T-T, Peng H-L. Preparation, characterization, and application of multiple stimuli-responsive rattle-type magnetic hollow molecular imprinted poly (ionic liquids) nanospheres $\left(\mathrm{Fe}_{3} \mathrm{O}_{4} @\right.$ void@PILMIP) for specific recognition of protein. Chem. Eng. J. 2018; 337: 722-732.

12. Dencheva N V, Oliveira F D, Braz J F, Denchev Z Z. Bovine serum albumin-imprinted magnetic poly(2pyrrolidone) microparticles for protein recognition. Eur. Polym. J. 2020; 122: 109375.

13. Lee M H, Lin C C, Thomas J L, Chan C K, Lin H Y. Epitope recognition of magnetic peptideimprinted chitosan composite nanoparticles for the extraction of CRISPR/dCas9a proteins from transfected cells.Nanotechnology 2021; 32: 18LT02.

14. Pasquardini L, Bossi A M. Molecularly imprinted polymers by epitope imprinting: a journey from molecular interactions to the available bioinformatics resources to scout for epitope templates. Anal Bioanal Chem 2021; 413: 6101-6115.

15. Qin Y P, Jia C, He X W, Li W Y, Zhang Y K. Thermosensitive Metal Chelation Dual-Template Epitope Imprinting Polymer Using Distillation-Precipitation Polymerization for Simultaneous Recognition of Human Serum Albumin and Transferrin. ACS Appl Mater Interfaces2018; 10: 9060-9068.

16. Kalecki J, Iskierko Z, Cieplak M, Sharma P S. Oriented Immobilization of Protein Templates: A New Trend in Surface Imprinting. ACS Sens 2020; 5: 3710-3720.

17. He P, Zhu H, Ma Y, Liu N, Niu X, Wei M, Pan J. Rational design and fabrication of surface molecularly imprinted polymers based on multi-boronic acid sites for selective capture glycoproteins. Chem. Eng. J. 2019; 367: 55-63.

18. Yang W, Zeng K, Liu J, Chen L, Wang M, Zhuo S, Ge X. Fabrication of inverse-opal lysozyme-imprinted polydopamine/polypyrrole microspheres with near-infrared-light-controlled release property. J Colloid Interface Sci 2019; 548: 37-47.

19. Xie X, Hu Q, Ke R, Zhen X, Bu Y, Wang S. Facile preparation of photonic and magnetic dual responsive protein imprinted nanomaterial for specific recognition of bovine hemoglobin. Chem. Eng. J. 2019; 371: $130-137$.

20. Kitayama Y, Isomura M. Gas-stimuli-responsive molecularly imprinted polymer particles with switchable affinity for target protein. Chem Commun 2018; 54: 2538-2541.

21. Wei Y, Zeng Q, Huang J, Guo X, Wang L, Wang L. Preparation of Gas-Responsive Imprinting Hydrogel and Their Gas-Driven Switchable Affinity for Target Protein Recognition. ACS Appl Mater Interfaces 2020; 12: 24363-24369.

22. Qian L, Liu W, Yang M, Nica V, Yang J, Liu H, Ning L, Zhang S, Zhang Q. Zwitterionic polymer chainassisted lysozyme imprinted core-shell carbon microspheres with enhanced recognition and selectivity. Talanta 2020; 217: 121085 . 
23. Han X, Han W, Zhang S, Liu Z, Fu G. PEGylation of protein-imprinted nanocomposites sandwiching CdTe quantum dots with enhanced fluorescence sensing selectivity. RSC Advances 2019; 9: 38165-38173.

24. Yang Z, Zhang Y, Ren J, Zhang Q, Zhang B. Cobalt-Iron Double Ion-Bovine Serum Albumin ChelationAssisted Thermo-Sensitive Surface-Imprinted Nanocage with High Specificity. ACS Appl Mater Interfaces 2021; 13: 34829-34842.

25. Hunyadi Murph S. E, Coopersmith K, Sessions H Jr, Brown M, Larsen G. Controlled Release of Hydrogen Isotopes from Hydride-Magnetic Nanomaterials. ACS Appl Mater Interfaces 2020; 12: 9478-9488.

26. Guo Z Y, Zhang C, Jiao R. W, Yao Q H, Ye T X, Chen X. Construction of Metal Hydrate-Based Amorphous Magnetic Nanosheets for Enhanced Protein Enrichment and Immobilization. ACS Appl Mater Interfaces 2021; 13: 37915-37923.

27. Zhang T, Huang B, Elzatahry A A, Alghamdi A, Yue Q, Deng Y. Synthesis of Podlike Magnetic Mesoporous Silica Nanochains for Use as Enzyme Support and Nanostirrer in Biocatalysis. ACS Appl Mater Interfaces 2020; 12: 17901-17908.

28. Su Y, Qiu B, Chang C, Li X, Zhang M, Zhou B, Yang Y. Separation of bovine hemoglobin using novel magnetic molecular imprinted nanoparticles. RSC Advances 2018; 8: 6192-6199.

29. Chen F, Mao M, Wang J, Liu J, Li F. A dual-step immobilization/imprinting approach to prepare magnetic molecular imprinted polymers for selective removal of human serum albumin. Talanta 2020; 209: 120509 .

30. Dinc M, Esen C, Mizaikoff B. Recent advances on core-shell magnetic molecularly imprinted polymers for biomacromolecules. Trends Analyt Chem 2019; 114: 202-217.

31. Li D, Tu T, Yang M, Xu C. Efficient preparation of surface imprinted magnetic nanoparticles using poly (2-anilinoethanol) as imprinting coating for the selective recognition of glycoprotein. Talanta2018; 184: 316-324.

32. Yang Z, Yang K, Cui Y, Shah T, Ahmad M, Zhang Q, Zhang B. Synthesis of surface imprinted polymers based on wrinkled flower-like magnetic graphene microspheres with favorable recognition ability for BSA.J. Mater. Sci. 2021; 74: 203-215.

33. Yang Z, Wang J, Shah T, Liu P, Ahmad M, Zhang Q, Zhang B. Development of surface imprinted heterogeneous nitrogen-doped magnetic carbon nanotubes as promising materials for protein separation and purification. Talanta 2021; 224: 121760.

34. Chang Y, Shu S H, Shih Y J, Chu C W, Ruaan R C, Chen W Y. Hemocompatible mixed-charge copolymer brushes of pseudozwitterionic surfaces resistant to nonspecific plasma protein fouling. Langmuir 2010; 26: 3522-30.

35. Cao Z, Jiang S. Super-hydrophilic zwitterionic poly(carboxybetaine) and amphiphilic non-ionic poly(ethylene glycol) for stealth nanoparticles. Nano Today 2012; 7: 404-413.

36. Wang Y, Zhou J, Gu H, Jia X, Su K, Zhang B, Zhang Q. Preparation of Anti-Nonspecific Adsorption Chitosan-Based Bovine Serum Albumin Imprinted Polymers with Outstanding Adsorption Capacity and Selective Recognition Ability Based on Magnetic Microspheres. Macromol Mater Eng 2019; 304: 1800731.

37. Li X, Zhang B, Tian L, Li W, Zhang H, Zhang Q. Improvement of recognition specificity of surface protein-imprinted magnetic microspheres by reducing nonspecific adsorption of competitors using 2methacryloyloxyethyl phosphorylcholine. Sens. Actuators B Chem.2015; 208: 559-568.

38. Wang Y, Ma Y, Zhou J, Su K, Zhang B, Zhang Q. $\mathrm{MnO}_{2}$ corolla-like magnetic molecularly imprinted microspheres with enhanced adsorption capacity and specificity recognition to bovine serum albumin. Chem. Eng. J. 2021; 405: 126655. 
39. Yang Z, Xu J, Wang J, Zhang Q, Zhang B. Design and preparation of self-driven BSA surface imprinted tubular carbon nanofibers and their specific adsorption performance. Chem. Eng. J. 2019; 373: 923-934.

40. Yang Z, Chen J, Yang K, Zhang Q, Zhang B. Preparation of BSA surface imprinted manganese dioxideloaded tubular carbon fibers with excellent specific rebinding to target protein. J Colloid Interface Sci2020; 570: $182-196$.

41. Zhou J, Wang Y, Bu J, Zhang B, Zhang Q. Ni ${ }^{(2+)}$-BSA Directional Coordination-Assisted Magnetic Molecularly Imprinted Microspheres with Enhanced Specific Rebinding to Target Proteins.ACS Appl Mater Interfaces 2019; 11: 25682-25690.

42. Zhang Y, Ren J, Yang Z, Ma Y, Zhang Q, Zhang B. Fabrication of Surface-Imprinted Magnetic Wrinkled Microspheres and Their Specific Adsorption of BSA. Ind. Eng. Chem. Res. 2021; 60: 11277-11288.

43. Qian L, Sun J, Hou C, Yang J, Li Y, Lei D, Yang M, Zhang S. Immobilization of BSA on ionic liquid functionalized magnetic Fe3O4 nanoparticles for use in surface imprinting strategy. Talanta2017; 168: 174-182.

44. Zhou J, Wang M, Zhang B, Zhang Q. Metal coordination assisted thermo-sensitive magnetic imprinted microspheres for selective adsorption and efficient elution of proteins. Colloids Surf. A Physicochem. Eng. Asp. 2021; 612: 125981.

45. Li X, Liu H, Deng Z, Chen W, Li T, Zhang Y, Zhang Z, He Y, Tan Z, Zhong S. PEGylated ThermoSensitive Bionic Magnetic Core-Shell Structure Molecularly Imprinted Polymers Based on Halloysite Nanotubes for Specific Adsorption and Separation of Bovine Serum Albumin.Polymers 2020; 12: 1-19. 